\title{
Dorsal Skin Microbial Assemblages in Free-ranging Hellbenders, Cryptobranchus Alleganiensis, Associated With Subspecies and Chronic Toe Lesions, but Not With Chytrid Fungal Infection.
}

Rebecca Hale Hardman ( $\nabla$ rhardman@utk.edu )

University of Tennessee Knoxville https://orcid.org/0000-0001-9083-8525

\section{Gary LeCleir}

The University of Tennessee Knoxville

\section{Benjamin Fitzpatrick}

The University of Tennessee Knoxville

Kelly J. Irwin

Arkansas Game and Fish Commission

William B. Sutton

Tennessee State University

Jeronimo G. da Silva Neto

The University of Tennessee Knoxville

Michael Freake

Lee University

Donald M. Walker

Middle Tennessee State University

Debra L. Miller

The University of Tennessee Knoxville

\section{Research Article}

Keywords: Hellbender, amphibian microbiome, salamander, wildlife disease, cutaneous lesions, chytrid, Bd

Posted Date: August 30th, 2021

DOI: https://doi.org/10.21203/rs.3.rs-827234/v1

License: (c) (i) This work is licensed under a Creative Commons Attribution 4.0 International License. Read Full License 


\section{Abstract}

Background

Skin microbiomes are important components of skin health and have been shown to contribute to immunity in amphibians, especially against the chytrid fungus, Batrachochytrium dendrobatidis $(B d)$. Hellbenders

(Cryptobranchus alleganiensis) are large aquatic amphibians (Order Caudata) native to the eastern United States that have experienced population declines of both the Ozark and eastern subspecies, C. a. bishopi and C. a. alleganiensis, respectively. In addition, ulcerative non-healing toe lesions have become increasingly prevalent in $C$. a. bishopi, in Arkansas (AR) where populations are now reduced to a single river. To evaluate the potential impacts of both chronic toe lesions and $B d$ on hellbender health, we compared dorsal skin microbial assemblages based on 16S rRNA gene amplicons between a declining Ozark hellbender population in Arkansas (AR) presenting lesions and a reference, recruiting, lesion-free, population of eastern hellbenders in eastern Tennessee (ETN). We further evaluated effects of mass and life stage across both subspecies, as well as toe lesion severity and $B d$ infection status within AR, to better understand the associations between microbiomes and disease in a wild salamander.

Results

We found skin of ETN hellbenders to have greater bacterial alpha diversity compared to AR, with this disparity decreasing as Hill number order increased. Conversely, within AR, animals with more severe lesions had decreased alpha diversity than those with mild lesions, which became more pronounced with increasing Hill number. Further, the average microbial assemblage structure differed between ETN and AR. Specifically, AR communities displayed increased beta diversity compared to those from ETN, which appeared to be linked to toe lesion severity. Neither size class (mass) nor $B d$ infection status had a significant effect on alpha or beta diversity. Taxonomic analysis revealed ETN to have greater OTU abundance of phylum Cyanobacteria 24.3\%) compared to AR (5.9\%); whereas AR had increased abundance of Proteobacteria (48.5\%), Firmicutes (9.1\%), and Synergistetes (1.5\%), in comparison to ETN (31.5\%, 2.6\%, 0.2\%, respectively).

Conclusions

Results demonstrate that eastern hellbenders of ETN have richer and less dispersed dorsal skin bacterial assemblages compared to Ozark hellbenders of AR. Furthermore, we suggest that increased severity of toe lesions may be linked to systemic changes resulting in skin microbial dysbiosis, independent of $B d$ infection. Although lesions remain to have an unknown etiology, this study is another step towards understanding skin bacterial microbiomes in hellbenders, and their potential associations with chronic disease.

\section{Background}

The skin is the first line of defense against invading pathogens and despite a highly developed immune system, thousands of bacterial species are resident on all vertebrate skin surfaces (Grice and Serge 2011). Increasing evidence reveals the critical role these microbial ecosystems (microbiomes) play in skin maintenance (Rodriquez Hoffman et al. 2015) and host immunity and health (de Vos and de Vos 2012; Shreiner et al. 2015; Arnolds and Lozupone 2016). Further, alterations in microbiomes are associated with important diseases in humans (Ilseung and Blaser 2012) and companion animals (Rodriguez Hoffman et al. 2015). As we continue to learn more about this complex ecosystem on host surfaces, we are discovering its importance as both an indicator of host health (Shreiner et al. 2015), and medical treatment tool (Kang et al. 2017). 
Evaluation of altered skin microbiomes may be especially important in amphibians. They rely on their skin for osmotic regulation, which is subsequently more permeable than other vertebrate hosts (Shoemaker and Nagy 1977). In addition, microbial community shifts may be major factors in amphibian host susceptibility to pathogens such as the amphibian chytrid fungus Batrachochytrium dendrobatidis $(B d)$, which is a fungal dermal pathogen responsible for the extinction of approximately 90 amphibian species worldwide (Scheele et al. 2019).

Intrinsic factors related to the amphibian host are important for colonization and maintenance of these skin bacterial communities (Jiménez and Sommer 2017). In apparently healthy amphibians, bacterial skin communities associate to species across different isolated ponds (McKenzie et al. 2011). Amphibian skin communities can also resist colonization from repeatedly applied bacterial skin washes (Küng et al. 2014). However, other studies reveal amphibian skin bacteria can in fact be shaped by the surrounding environment. Amphibian skin microbial community metrics have been linked to climate and biosphere (Kueneman et al. 2019; Jani 2019), environmental substrate (Fitzpatrick and Allison 2014), and microhabitat type (Bletz et al. 2017a). This dynamic relationship between amphibian host and environment on skin microbiome composition is an important consideration during an assessment of wild amphibian health (Jani and Briggs 2018; Bernardo-Cravo et al. 2020). Associations between environmental degradation and altered host-associated microbial communities are documented in other wildlife species such as black howler monkeys (Amato et al. 2013) and corals (Krediet et al. 2013). In corals particularly, these host-associated microbial community changes have been further linked to decreased coral immunity (Glasl et al. 2016).

Hellbenders, Cryptobranchus alleganiensis, are fully aquatic riverine amphibians (Order Caudata) of the eastern United States and one of only three species of giant salamanders (family: Cryptobranchidae). Populations of both subspecies, the Ozark hellbender, $C$. a. bishopi, and eastern hellbender, $C$. a. alleganiensis, have experienced considerable declines since the early 1990's resulting in listing of the Ozark hellbender as endangered in 2011 (USFWS 2011). Declines in both subspecies are linked to habitat degradation and sedimentation from eroding stream banks (Humphries and Pauley 2005; Hopkins and DuRant 2011; USFWS 2011, Pugh et al. 2015), but disease is also hypothesized to play a role (Hardman et al. 2020a,b; Nickerson et al. 2011). In Ozark hellbenders of Arkansas (AR), over $93 \%$ of individuals had progressive distal limb lesions that ranged from toe swelling to ulceration and digital necrosis (Hardman et al. 2020a). Toe lesions are documented to occur and worsen over several years. There is no identified etiology but disease is likely manifesting from a multifactorial process (Hardman et al. 2020a). These lesions may be linked to environmental factors, although, host and infectious factors are hypothesized to contribute (Hardman et al. 2020a; Nickerson et al. 2011). For example, Ozark hellbenders have been identified to have decreased allelic diversity of genes important for adaptive immunity (Hernández-Gómez et al. 2018). Changes in the skin microbiome may help identify important aspects of disease pathophysiology. In humans, skin microbial community alterations can be associated with chronic skin wounds (Grice and Segre 2012; Scales and Huffnagle 2012; Pang et al. 2019) and chronic skin diseases such as psoriasis (Thio 2018). However, it is still speculative as to how changes in skin immunity may be associated with chronic toe lesions observed in wild hellbender populations.

Although there has been extensive research on how skin microbiomes may modulate susceptibility of amphibian hosts to Bd infection (Becker and Harris 2010; Bletz et al. 2017a; Jani and Briggs 2018; Bernardo-Cravo et al. 2020), there is a paucity of research on how these surface communities may be related to wound healing. Only one study has evaluated foot lesions in a small sample of Ozark hellbenders and found bacterial assemblages on toe lesions were richer and had differential abundance of core taxa when compared to the dorsal skin (Hernández-Gómez et al. 2017b). It will be important to also understand how lesion presence and severity may be associated with overall changes in the skin microbiome to better mitigate this disease. Furthermore, hellbenders are long-lived animals ( $>20$ 
years) and present an opportunity for us to understand the long-term effect of chronic disease in a wild amphibian species, particularly related to the association between chronic non-healing wounds and skin microbiomes.

Therefore, we evaluated dorsal skin bacterial assemblages of hellbender populations from two rivers of different conservation status: a stable population of eastern hellbenders in eastern Tennessee (ETN) and a declining and diseased population of Ozark hellbenders in Arkansas (AR). We further evaluated whether variation within the unhealthy AR population could be explained by toe lesion severity or $B d$ infection status. Lastly we evaluated how life stage and mass could help explain any observed variation.

\section{Results}

We sampled 63 individuals, 37 from AR and 26 from ETN (Fig. 1). We recovered 2,804,796 sequences assigned to 911 phylotypes after sequence processing in MOTHUR. Sequence counts per sample ranged from 3 to 379,352 .

Subsampling and quality control resulted in subsequent removal of 25 samples with less than 4000 sequences, leaving 40 samples remaining for analysis ( 15 AR and 25 ETN). Within the 15 AR samples, two were from individuals with mild toe lesions, seven with moderate lesions, and six with severe lesions. The 15 AR samples were also screened for the presence of $B d$, using quantitative PCR (qPCR). This screening process identified six individuals that were $B d$ positive within the AR samples. We encountered and sampled juveniles and adults in ETN (nine and 16, respectively), but only adults were sampled from AR. Mass and subsequent size class data were available for all individuals sampled for both subspecies with eight small and eight medium adults in ETN and six medium and nine large adults in AR. Mass ranged from $3 g-807 g$ with a median of $320 \mathrm{~g}$.

\section{Subspecies, size, and life stage}

Eastern hellbenders of ETN had greater relativized richness (Hill number $q=0)$ than Ozark hellbenders of AR $(p<$ 0.001; Fig. 2, Table 1). Hill numbers for exponential Shannon Diversity $(q=1)$ and inverse Simpson $(q=2)$ were also greater for ETN compared to AR hellbender skin but were not significant $(p=0.24$ and 0.53 , respectively; Fig. 2 , Table 1). Average assemblages significantly differed between subspecies via both Sorenson Index $\left(F_{1,39}=9.7, p<\right.$ $\left.0.001, \mathrm{R}^{2}=0.21\right)$ and Morisita-Horn $\left(F_{1,39}=12.5, p<0.001, \mathrm{R}^{2}=0.25\right)$. Non-metric Multidimensional Scaling (NMDS) ordination plots highlighted distinct grouping between subspecies and displayed increased beta diversity of AR hellbender skin assemblages compared to ETN (Fig. 4). This was confirmed via beta dispersion analysis (betadisper, Morisita-Horn, $p=0.01$; Sorenson Index, $p=0.002$ ). Mass was not predictive of alpha diversity for hellbender subspecies (Hill q =0: $p=0.48,1: p=0.90,2: p=0.83$; Table 1). Similarly, adult size class was not predictive of average assembly structure of hellbender subspecies (Sorenson Index: $p=0.81$ Morisita-Horn: $p=0.95$ ). Within ETN, no effect of life stage was seen for alpha diversity (Hill $q=0: p=0.19,1: p=0.19,2: p=0.77 ;$ Table 1). Average assemblage structure of juvenile skin differed from adults using the Sorensen Index $\left(F_{1,24}=1.8, p=0.03, \mathrm{R}^{2}=0.07\right.$,

Fig. 4), but not Morisita-Horn $\left(F_{1,24}=0.4, p=0.92, \mathrm{R}^{2}=0.02\right.$, Fig. 4). To remove potential effects of juvenile sampling on subspecies comparisons, we reran all subspecies analyses with only adults from ETN $(n=16)$ and found that the previously mentioned relationships remained unchanged (see Table 1, Fig. 2,4). 
Table 1

Average Hill numbers (orders $q=0,1,2$ ) for each factor comparison of skin microbial assemblage alpha diversity from the dorsum of eastern hellbenders (Cryptobranchus alleganiensis alleganiensis) of eastern

Tennessee (ETN) and Ozark hellbenders (Cryptobranchus alleganiensis bishopi) of Arkansas (AR). Comparison groups were as follows: subspecies (ETN, AR), life stage within ETN only (juvenile, adult), toe lesion severity (mild, moderate, severe) within AR only, and Batrachochytrium dendrobatidis ( $B d$ ) infection status within AR only $(B d+, B d-)$.

\begin{tabular}{|c|c|c|c|c|c|c|c|}
\hline \multirow[t]{2}{*}{ Factor } & \multirow[t]{2}{*}{$\mathbf{n}$} & \multicolumn{6}{|c|}{ Hill Number (avg +/- SD) } \\
\hline & & $q=0$ & & $q=1$ & & $q=2$ & \\
\hline \multicolumn{8}{|l|}{ Subspecies } \\
\hline ETN & 25 & 140 & $+/-17.8$ & 45.4 & $+/-13.51$ & 19.0 & $+/-8.92$ \\
\hline AR & 15 & 111.4 & $+/-23.9$ & 40.3 & $+/-12.3$ & 20.1 & $+/-8.49$ \\
\hline \multicolumn{8}{|c|}{ Life Stage (ETN Only) } \\
\hline Juvenile & 9 & 133.0 & $+/-24.3$ & 44.3 & $+/-18.0$ & 19.8 & $+/-12.5$ \\
\hline Adult & 16 & 144.0 & $+/-12.1$ & 46.0 & $+/-10.9$ & 18.6 & $+/-6.6$ \\
\hline \multicolumn{8}{|c|}{ Lesion Severity (AR Only) } \\
\hline Mild & 2 & 132.4 & $+/-0.9$ & 60.4 & $+/-3.5$ & 36.0 & $+/-0.2$ \\
\hline Moderate & 7 & 104.3 & $+/-23.7$ & 34.8 & $+/-5.4$ & 16.5 & $+/-3.7$ \\
\hline Severe & 6 & 112.7 & $+/-26.1$ & 39.9 & $+/-13.5$ & 19.0 & $+/-8.0$ \\
\hline \multicolumn{8}{|c|}{ Bd Infection Status (AR Only) } \\
\hline$B d+$ & 6 & 111.6 & +/- 19.2 & 39.0 & $+/-10.6$ & 18.4 & $+/-9.1$ \\
\hline$B d-$ & 9 & 111.2 & $+/-27.8$ & 41.1 & $+/-13.9$ & 21.2 & $+/-8.4$ \\
\hline
\end{tabular}

\section{Lesion severity and Bd infection within AR populations}

Within AR, disparity between mild and moderate/severe lesion groups increased with increasing q order. Dorsal skin assemblages from animals with mild toe lesions had greater relativized richness compared to those with both moderate $(p=0.18)$, and severe $(p=0.31)$, and had significantly greater exponential Shannon Diversity $(q=1 ; p=$ 0.009 and 0.024 , respectively) and inverse Simpson ( $q=2) ; p=0.010$ and 0.017 , respectively) (Fig. 3, Table 1). Average assemblage structure did not differ between lesion severity groups especially when relative abundance was not considered (Sorenson Index, $F_{2,14}=0.85, p=0.59, \mathrm{R}^{2}=0.13$; Morisita-Horn, $F_{2,14}=1.86, p=0.08, \mathrm{R}^{2}=0.24$; Fig. 5). Beta dispersion increased with increasing lesion severity for both Morisita-Horn and Sorenson Indices but was not significant ( $p=0.09$ and 0.12 , respectively; Fig. 5).

There was no effect of $B d$ infection status on measures of alpha diversity (Table 1, Fig. 3) or average assemblage structure (Sorenson Index, $F_{1,14}=0.73, p=0.68, \mathrm{R}^{2}=0.05$; Morisita Horn, $F_{1,14}=0.70, p=0.56, \mathrm{R}^{2}=0.05$; Fig. 5). There was no difference in beta dispersion between $B d$ infection-status groups of AR individuals for both Morisita-Horn and Sorenson Indices ( $p=0.80$ and 0.42 , respectively; Fig. 4)

\section{Bacterial Taxonomic Analysis}


We identified 468 phylotypes represented by 28 bacterial phyla during sequence processing. Ozark AR and eastern ETN hellbenders shared 312 out of 468 phylotypes, with 121 unique to ETN and 35 unique to AR. ETN had on average greater phylotype abundance of phylum Cyanobacteria (24.3\%), Acidobacteria (2.7\%), and Armatimonadetes (1.0\%) compared to $\operatorname{AR}(5.9 \%, 1.1 \%$, and $0.3 \%$, respectively). Hellbenders of AR had a greater average relative abundance of Proteobacteria (48.5\%), Firmicutes (9.1\%), and Synergistetes (1.5\%), in comparison to ETN with (31.5\%, 2.6\%, 0.2\%, respectively) (Fig. 6, Table 2). For indicator species analysis, 65 phylotypes were considered indicative of ETN dorsal skin compared to 34 for AR (Table 3). There were three top indicators (IndVal Index stat >0.9) for ETN which belonged to genus Ferruginibacter, an unclassified order of Cyanobacteria (incertae sedis), and an unclassified group of Verrucomicrobia (subdivision 3). Only one top phylotype (genus Limnohabitans) was identified for AR (Table 3). 
Table 2

Average \% phylum abundance of the 28 bacterial phlya identified from 1000 sequence subsamples of $16 \mathrm{~S}$ sequencing from dorsal skin swabs of eastern hellbenders (Cryptobranchus alleganiensis alleganiensis) of eastern Tennessee (ETN) and Ozark hellbenders (Cryptobranchus alleganiensis bishopi) of Arkansas (AR). AR hellbenders are further grouped by toe lesion severity (mild, moderate serve) and Batrachochytrium dendrobatidis ( $B d$ ) infection status $(B d+, B d)$. ETN hellbenders are further grouped by life stage (juvenile, adult).

\begin{tabular}{|c|c|c|c|c|c|c|c|c|c|}
\hline \multirow[t]{2}{*}{ Phylum } & \multicolumn{3}{|c|}{ ETN Eastern Hellbender } & \multicolumn{6}{|c|}{ AR Ozark Hellbender } \\
\hline & All & Juvenile & $\begin{array}{l}\text { Adult } \\
\text { ETN }\end{array}$ & All & Mild & Moderate & Severe & $B d-$ & $B d+$ \\
\hline $\begin{array}{l}\text { Bacteria } \\
\text { unclassified }\end{array}$ & 7.54 & 6.93 & 7.88 & 5.91 & 7.65 & 7.51 & 3.47 & 5.37 & 6.73 \\
\hline Proteobacteria & 31.49 & 31.51 & 31.48 & 48.48 & 41.00 & 56.81 & 41.25 & 48.59 & 48.32 \\
\hline Cyanobacteria & 24.34 & 24.49 & 24.25 & 5.89 & 8.25 & 4.31 & 6.95 & 6.58 & 4.87 \\
\hline Planctomycetes & 4.47 & 3.41 & 5.07 & 2.65 & 5.45 & 2.96 & 1.35 & 1.63 & 4.17 \\
\hline Bacteroidetes & 11.96 & 13.14 & 11.29 & 11.91 & 16.65 & 7.79 & 15.15 & 10.76 & 13.65 \\
\hline Verrucomicrobia & 4.12 & 3.11 & 4.69 & 2.17 & 2.75 & 2.51 & 4.02 & 3.52 & 2.58 \\
\hline $\begin{array}{l}\text { Deinococcus- } \\
\text { Thermus }\end{array}$ & 1.63 & 2.56 & 1.11 & 3.15 & 0.80 & 0.30 & 0.10 & 0.27 & 0.32 \\
\hline OD1 & 0.72 & 0.90 & 0.62 & 0.29 & 0.55 & 1.37 & 0.67 & 1.20 & 0.65 \\
\hline TM7 & 0.98 & 0.93 & 1.01 & 0.98 & 6.35 & 5.24 & 3.67 & 4.58 & 5.03 \\
\hline Actinobacteria & 4.54 & 3.63 & 5.04 & 4.76 & 2.15 & 1.06 & 0.85 & 1.09 & 1.17 \\
\hline Acidobacteria & 2.69 & 2.12 & 3.01 & 1.12 & 5.20 & 1.76 & 1.65 & 1.80 & 2.73 \\
\hline Armatimonadetes & 1.04 & 1.07 & 1.03 & 0.26 & 0.50 & 0.17 & 0.28 & 0.24 & 0.28 \\
\hline Firmicutes & 2.58 & 3.59 & 2.01 & 9.13 & 0.85 & 5.01 & 16.70 & 10.61 & 6.92 \\
\hline Chloroflexi & 0.60 & 0.18 & 0.17 & 0.39 & 0.40 & 0.44 & 0.32 & 0.24 & 0.60 \\
\hline WS3 & 0.17 & 0.58 & 0.61 & 0.05 & 0.05 & 0.00 & 0.10 & 0.02 & 0.08 \\
\hline Gemmatimonadetes & 0.14 & 0.06 & 0.19 & 0.08 & 0.30 & 0.06 & 0.03 & 0.09 & 0.07 \\
\hline Nitrospira & 0.13 & 0.14 & 0.13 & 0.07 & 0.15 & 0.06 & 0.07 & 0.10 & 0.03 \\
\hline Fusobacteria & 0.14 & 0.20 & 0.11 & 0.48 & 0.05 & 0.10 & 1.07 & 0.67 & 0.20 \\
\hline Synergistetes & 0.16 & 0.46 & 0.00 & 1.49 & 0.00 & 1.77 & 1.67 & 1.76 & 1.10 \\
\hline Chlamydiae & 0.22 & 0.22 & 0.21 & 0.13 & 0.50 & 0.09 & 0.05 & 0.10 & 0.17 \\
\hline Spirochaetes & 0.14 & 0.40 & 0.00 & 0.04 & 0.10 & 0.06 & 0.00 & 0.06 & 0.02 \\
\hline BRC1 & 0.03 & 0.29 & 0.05 & 0.01 & 0.05 & 0.00 & 0.00 & 0.01 & 0.00 \\
\hline Lentisphaerae & 0.14 & 0.04 & 0.03 & 0.36 & 0.00 & 0.39 & 0.45 & 0.54 & 0.08 \\
\hline SR1 & 0.02 & 0.01 & 0.02 & 0.04 & 0.00 & 0.09 & 0.00 & 0.00 & 0.10 \\
\hline Chlorobi & 0.00 & 0.00 & 0.01 & 0.08 & 0.25 & 0.06 & 0.05 & 0.06 & 0.12 \\
\hline
\end{tabular}




\begin{tabular}{|llllllllll|}
\hline Phylum & \multicolumn{1}{l}{ ETN Eastern Hellbender } & \multicolumn{3}{ll}{ AR Ozark Hellbender } & & & \\
\hline Deferribacteres & 0.00 & 0.00 & 0.00 & 0.04 & 0.00 & 0.06 & 0.03 & 0.06 & 0.02 \\
\hline Elusimicrobia & 0.00 & 0.00 & 0.00 & 0.04 & 0.00 & 0.03 & 0.07 & 0.07 & 0.00 \\
\hline Fibrobacteres & 0.01 & 0.02 & 0.00 & 0.00 & 0.00 & 0.00 & 0.00 & 0.00 & 0.00 \\
\hline
\end{tabular}


Table 3

List of indicator taxa based on indicator species analysis significantly associated with dorsal skin of hellbenders within each comparison group based on subspecies (eastern hellbenders (Cryptobranchus alleganiensis alleganiensis) of eastern Tennessee (ETN) and Ozark hellbenders (Cryptobranchus alleganiensis bishopi) of Arkansas (AR)), life stage within ETN (juvenile vs adult), lesion severity within AR (mild vs moderate vs severe) and Batrachochytrium dendrobatidis ( $B d)$ infection status $(B d+v s B d-)$. No taxa were identified as significantly associated with animals with severe lesions. Taxa with a stat $>0.9$ were considered top indicator taxa and are noted in bold.

$\begin{array}{lllll}\text { Factor } & \text { Group } & \text { stat } & p_{\text {value }} & \text { Phylum }\end{array}$

Subspecies

\begin{tabular}{|c|c|c|c|c|}
\hline ETN & 0.954 & 0.001 & Bacteroidetes & Ferruginibacter \\
\hline ETN & 0.945 & 0.001 & $\begin{array}{l}\text { Cyanobacteria } \\
\text { Chloroplast }\end{array}$ & $\begin{array}{l}\text { Cyanobacteria order incertae } \\
\text { sedis unclassified }\end{array}$ \\
\hline ETN & 0.915 & 0.001 & Verrucomicrobia & 3 genus incertae sedis \\
\hline ETN & 0.882 & 0.001 & $\begin{array}{l}\text { Cyanobacteria } \\
\text { Chloroplast }\end{array}$ & Bacillariophyta \\
\hline ETN & 0.879 & 0.001 & Verrucomicrobia & Opitutus \\
\hline ETN & 0.874 & 0.001 & Acidobacteria & Gp3 \\
\hline ETN & 0.869 & 0.001 & Armatimonadetes & $\begin{array}{l}\text { Armatimonas Armatimonadetes } \\
\text { gp } 1\end{array}$ \\
\hline ETN & 0.867 & 0.001 & Actinobacteria & Actinobacteria unclassified \\
\hline ETN & 0.864 & 0.001 & Bacteroidetes & Sphingobacteriales unclassified \\
\hline ETN & 0.862 & 0.001 & $\begin{array}{l}\text { Cyanobacteria } \\
\text { Chloroplast }\end{array}$ & $G p l$ \\
\hline ETN & 0.855 & 0.001 & Proteobacteria & Myxococcales unclassified \\
\hline ETN & 0.854 & 0.001 & Bacteroidetes & Ohtaekwangia \\
\hline ETN & 0.846 & 0.001 & OD1 & OD1 genus incertae sedis \\
\hline ETN & 0.841 & 0.001 & Proteobacteria & Betaproteobacteria unclassified \\
\hline ETN & 0.838 & 0.001 & $\begin{array}{l}\text { Cyanobacteria } \\
\text { Chloroplast }\end{array}$ & GpVI \\
\hline ETN & 0.836 & 0.001 & Proteobacteria & Proteobacteria unclassified \\
\hline ETN & 0.833 & 0.001 & Actinobacteria & Acidimicrobiales unclassified \\
\hline ETN & 0.830 & 0.002 & Proteobacteria & $\begin{array}{l}\text { Alphaproteobacteria } \\
\text { unclassified }\end{array}$ \\
\hline ETN & 0.826 & 0.001 & Proteobacteria & Hyphomicrobium \\
\hline ETN & 0.826 & 0.001 & Bacteroidetes & Flammeovirgaceae unclassified \\
\hline ETN & 0.825 & 0.001 & Chloroflexi & Ktedonobacterales unclassified \\
\hline ETN & 0.824 & 0.001 & Proteobacteria & Cellvibrio \\
\hline ETN & 0.819 & 0.001 & Proteobacteria & Rhodospirillales unclassified \\
\hline
\end{tabular}




\begin{tabular}{|c|c|c|c|c|c|}
\hline \multirow[t]{2}{*}{ Factor } & Group & stat & $\begin{array}{l}p \\
\text { value }\end{array}$ & Phylum & Genus \\
\hline & ETN & 0.807 & 0.002 & $\begin{array}{l}\text { Deinococcus- } \\
\text { Thermus }\end{array}$ & Deinobacterium \\
\hline & ETN & 0.803 & 0.007 & Verrucomicrobia & $\begin{array}{l}\text { Spartobacteria genera incertae } \\
\text { sedis }\end{array}$ \\
\hline & ETN & 0.802 & 0.015 & Planctomycetes & Planctomycetaceae unclassified \\
\hline & ETN & 0.802 & 0.001 & Proteobacteria & Phaselicystis \\
\hline & ETN & 0.800 & 0.001 & Proteobacteria & Bradyrhizobiaceae unclassified \\
\hline & ETN & 0.790 & 0.01 & Proteobacteria & $\begin{array}{l}\text { Gammaproteobacteria } \\
\text { unclassified }\end{array}$ \\
\hline & ETN & 0.784 & 0.02 & Acidobacteria & Gp16 \\
\hline & ETN & 0.781 & 0.033 & Planctomycetes & Planctomyces \\
\hline & ETN & 0.781 & 0.021 & Acidobacteria & Gp4 \\
\hline & ETN & 0.777 & 0.01 & Verrucomicrobia & Verrucomicrobia unclassified \\
\hline & ETN & 0.775 & 0.003 & $\begin{array}{l}\text { Deinococcus- } \\
\text { Thermus }\end{array}$ & Deinococcaceae unclassified \\
\hline & ETN & 0.765 & 0.038 & Planctomycetes & Gemmata \\
\hline & ETN & 0.762 & 0.002 & Armatimonadetes & $\begin{array}{l}\text { Chthonomonas } \\
\text { Armatimonadetes gp3 }\end{array}$ \\
\hline & ETN & 0.753 & 0.005 & WS3 & WS3 genus incertae sedis \\
\hline & ETN & 0.751 & 0.011 & Proteobacteria & Bradyrhizobium \\
\hline & ETN & 0.748 & 0.041 & Proteobacteria & Deltaproteobacteria unclassified \\
\hline & ETN & 0.748 & 0.001 & Proteobacteria & Rhizobacter \\
\hline & ETN & 0.741 & 0.001 & Proteobacteria & Acetobacteraceae unclassified \\
\hline & ETN & 0.721 & 0.005 & Bacteroidetes & Arcicella \\
\hline & ETN & 0.721 & 0.001 & Actinobacteria & Conexibacter \\
\hline & ETN & 0.701 & 0.005 & Actinobacteria & Pseudonocardia \\
\hline & ETN & 0.693 & 0.003 & Proteobacteria & Undibacterium \\
\hline & ETN & 0.693 & 0.002 & Acidobacteria & Gp1 \\
\hline & ETN & 0.683 & 0.014 & Actinobacteria & Solirubrobacterales unclassified \\
\hline & ETN & 0.670 & 0.02 & Proteobacteria & Kofleria \\
\hline & ETN & 0.662 & 0.037 & Proteobacteria & Anaeromyxobacter \\
\hline & ETN & 0.658 & 0.009 & Proteobacteria & Polyangiaceae unclassified \\
\hline & ETN & 0.646 & 0.021 & Actinobacteria & Nocardioidaceae unclassified \\
\hline
\end{tabular}




\begin{tabular}{|c|c|c|c|c|c|}
\hline Factor & Group & stat & $\begin{array}{l}\mathrm{p} \\
\text { value }\end{array}$ & Phylum & Genus \\
\hline & ETN & 0.645 & 0.018 & $\begin{array}{l}\text { Cyanobacteria } \\
\text { Chloroplast }\end{array}$ & $\begin{array}{l}\text { Cyanobacteria Chloroplast } \\
\text { unclassified }\end{array}$ \\
\hline & ETN & 0.638 & 0.014 & Bacteroidetes & Rudanella \\
\hline & ETN & 0.632 & 0.013 & Bacteroidetes & Fibrella \\
\hline & ETN & 0.632 & 0.012 & Acidobacteria & $G p 2$ \\
\hline & ETN & 0.632 & 0.007 & Acidobacteria & Gp5 \\
\hline & ETN & 0.631 & 0.02 & Actinobacteria & Marmoricola \\
\hline & ETN & 0.620 & 0.026 & Acidobacteria & Geothrix \\
\hline & ETN & 0.609 & 0.033 & Proteobacteria & Byssovorax \\
\hline & ETN & 0.600 & 0.05 & Planctomycetes & Schlesneria \\
\hline & ETN & 0.600 & 0.021 & Chloroflexi & Ktedonobacter \\
\hline & ETN & 0.566 & 0.029 & Acidobacteria & Holophagaceae unclassified \\
\hline & ETN & 0.566 & 0.025 & Proteobacteria & Rhodoferax \\
\hline & ETN & 0.562 & 0.048 & Actinobacteria & Sporichthya \\
\hline & ETN & 0.529 & 0.048 & Actinobacteria & Phycicoccus \\
\hline & AR & 0.925 & 0.001 & Proteobacteria & Limnohabitans \\
\hline & AR & 0.894 & 0.002 & Proteobacteria & Acinetobacter \\
\hline & AR & 0.874 & 0.001 & Bacteroidetes & Cytophagaceae unclassified \\
\hline & $A R$ & 0.845 & 0.006 & Proteobacteria & Burkholderiales unclassified \\
\hline & AR & 0.840 & 0.015 & Proteobacteria & Aeromonas \\
\hline & AR & 0.836 & 0.008 & $\begin{array}{l}\text { Deinococcus- } \\
\text { Thermus }\end{array}$ & Deinococcus \\
\hline & AR & 0.834 & 0.002 & Proteobacteria & Rhodobacteraceae unclassified \\
\hline & AR & 0.832 & 0.002 & Bacteroidetes & Flavobacterium \\
\hline & AR & 0.831 & 0.01 & Proteobacteria & Sphingomonas \\
\hline & $A R$ & 0.825 & 0.004 & Verrucomicrobia & Luteolibacter \\
\hline & AR & 0.806 & 0.006 & Actinobacteria & Microbacteriaceae unclassified \\
\hline & AR & 0.800 & 0.001 & Proteobacteria & Vogesella \\
\hline & AR & 0.773 & 0.001 & Proteobacteria & Roseomonas \\
\hline & AR & 0.760 & 0.001 & Bacteroidetes & Cryomorphaceae unclassified \\
\hline & AR & 0.758 & 0.037 & Verrucomicrobia & $\begin{array}{l}\text { Verrucomicrobiaceae } \\
\text { unclassified }\end{array}$ \\
\hline
\end{tabular}




\begin{tabular}{|c|c|c|c|c|c|}
\hline \multirow[t]{2}{*}{ Factor } & Group & stat & $\begin{array}{l}\mathrm{p} \\
\text { value }\end{array}$ & Phylum & Genus \\
\hline & AR & 0.740 & 0.041 & Proteobacteria & $\begin{array}{l}\text { Xanthomonadaceae } \\
\text { unclassified }\end{array}$ \\
\hline & AR & 0.728 & 0.003 & Bacteroidetes & Chryseobacterium \\
\hline & AR & 0.727 & 0.044 & Bacteroidetes & Flavobacteriaceae unclassified \\
\hline & $A R$ & 0.720 & 0.003 & Actinobacteria & Coriobacteriaceae unclassified \\
\hline & AR & 0.699 & 0.002 & Proteobacteria & Methylobacter \\
\hline & AR & 0.696 & 0.016 & Proteobacteria & Haliea \\
\hline & $A R$ & 0.664 & 0.005 & Proteobacteria & $\begin{array}{l}\text { Pseudomonadaceae } \\
\text { unclassified }\end{array}$ \\
\hline & $A R$ & 0.657 & 0.003 & Proteobacteria & Polynucleobacter \\
\hline & $A R$ & 0.642 & 0.015 & Firmicutes & Exiguobacterium \\
\hline & AR & 0.627 & 0.02 & Proteobacteria & Dechloromonas \\
\hline & $A R$ & 0.626 & 0.005 & Verrucomicrobia & Opitutae unclassified \\
\hline & AR & 0.608 & 0.037 & Firmicutes & Planococcaceae unclassified \\
\hline & $A R$ & 0.600 & 0.035 & Firmicutes & Clostridium XI \\
\hline & $A R$ & 0.598 & 0.028 & Firmicutes & Clostridiales unclassified \\
\hline & $A R$ & 0.563 & 0.019 & Chlorobi & Ignavibacterium \\
\hline & $A R$ & 0.516 & 0.016 & Proteobacteria & Methylosoma \\
\hline & AR & 0.516 & 0.015 & Proteobacteria & Silanimonas \\
\hline & AR & 0.500 & 0.036 & $\begin{array}{l}\text { Cyanobacteria } \\
\text { Chloroplast }\end{array}$ & Cryptomonadaceae \\
\hline & AR & 0.488 & 0.039 & Proteobacteria & Phyllobacteriaceae unclassified \\
\hline \multicolumn{6}{|c|}{ Life Stage (ETN Only) } \\
\hline & Adult & 0.824 & 0.004 & Planctomycetes & Planctomyces \\
\hline & Adult & 0.811 & 0.033 & Verrucomicrobia & 3 genus incertae sedis \\
\hline & Adult & 0.796 & 0.049 & Actinobacteria & Actinomycetales unclassified \\
\hline & Adult & 0.792 & 0.028 & Gemmatimonadetes & Gemmatimonas \\
\hline & Adult & 0.784 & 0.039 & Planctomycetes & Zavarzinella \\
\hline & Adult & 0.777 & 0.019 & Actinobacteria & \\
\hline & & & & & Solirubrobacterales unclassified \\
\hline & Adult & 0.775 & 0.04 & Proteobacteria & Betaproteobacteria unclassified \\
\hline & Adult & 0.744 & 0.029 & Proteobacteria & Undibacterium \\
\hline
\end{tabular}




\begin{tabular}{|c|c|c|c|c|c|}
\hline \multirow[t]{13}{*}{ Factor } & Group & stat & $\begin{array}{l}\mathrm{p} \\
\text { value }\end{array}$ & Phylum & Genus \\
\hline & Adult & 0.707 & 0.026 & Proteobacteria & Haliea \\
\hline & Adult & 0.707 & 0.024 & Proteobacteria & Inhella \\
\hline & Juvenile & 0.867 & 0.002 & $\begin{array}{l}\text { Deinococcus- } \\
\text { Thermus }\end{array}$ & Deinococcus \\
\hline & Juvenile & 0.833 & 0.013 & Proteobacteria & Acinetobacter \\
\hline & Juvenile & 0.820 & 0.026 & Proteobacteria & Sphingomonas \\
\hline & Juvenile & 0.809 & 0.022 & Proteobacteria & Oxalobacteraceae unclassified \\
\hline & Juvenile & 0.785 & 0.04 & Proteobacteria & Methylobacterium \\
\hline & Juvenile & 0.760 & 0.007 & Proteobacteria & Delftia \\
\hline & Juvenile & 0.680 & 0.041 & $\begin{array}{l}\text { Cyanobacteria } \\
\text { Chloroplast }\end{array}$ & Chlorophyta \\
\hline & Juvenile & 0.624 & 0.039 & Proteobacteria & Caulobacter \\
\hline & Juvenile & 0.577 & 0.036 & Bacteroidetes & Chitinophaga \\
\hline & Juvenile & 0.577 & 0.035 & Bacteroidetes & Cytophaga \\
\hline \multicolumn{6}{|c|}{$\begin{array}{l}\text { Lesion Severity (AR } \\
\text { Only) }\end{array}$} \\
\hline & Mild & 0.953 & 0.009 & Verrucomicrobia & Opitutus \\
\hline & Mild & 0.947 & 0.011 & Bacteroidetes & Emticicia \\
\hline & Mild & 0.920 & 0.021 & Bacteroidetes & Ferruginibacter \\
\hline & Mild & 0.906 & 0.036 & Proteobacteria & Myxococcales unclassified \\
\hline & Mild & 0.901 & 0.008 & Planctomycetes & Zavarzinella \\
\hline & Mild & 0.892 & 0.026 & Bacteroidetes & Fluviicola \\
\hline & Mild & 0.877 & 0.01 & Gemmatimonadetes & Gemmatimonas \\
\hline & Mild & 0.874 & 0.046 & Chlamydiae & Parachlamydiaceae unclassified \\
\hline & Mild & 0.873 & 0.025 & Verrucomicrobia & Verrucomicrobia unclassified \\
\hline & Mild & 0.862 & 0.04 & Proteobacteria & Inhella \\
\hline & Mild & 0.856 & 0.008 & Bacteroidetes & Sphingobacteriales unclassified \\
\hline & Mild & 0.849 & 0.023 & Proteobacteria & Steroidobacter \\
\hline & Mild & 0.846 & 0.041 & Acidobacteria & $G p 7$ \\
\hline & Mild & 0.846 & 0.036 & Proteobacteria & Desulfocapsa \\
\hline & Mild & 0.846 & 0.029 & Proteobacteria & Hyphomicrobiaceae unclassified \\
\hline & Mild & 0.846 & 0.022 & Proteobacteria & Haliea \\
\hline
\end{tabular}




\begin{tabular}{|c|c|c|c|c|c|}
\hline Factor & Group & stat & $\begin{array}{l}\mathrm{p} \\
\text { value }\end{array}$ & Phylum & Genus \\
\hline & Mild & 0.837 & 0.047 & Chlorobi & Ignavibacterium \\
\hline & Mild & 0.833 & 0.034 & Proteobacteria & Comamonadaceae unclassified \\
\hline & Mild & 0.832 & 0.023 & Verrucomicrobia & Verrucomicrobium \\
\hline & Mild & 0.828 & 0.018 & Proteobacteria & $\begin{array}{l}\text { Xanthomonadaceae } \\
\text { unclassified }\end{array}$ \\
\hline & Mild & 0.826 & 0.021 & Proteobacteria & Rhodocyclaceae unclassified \\
\hline & Mild & 0.817 & 0.024 & Verrucomicrobia & 3 genus incertae sedis \\
\hline & Mild & 0.816 & 0.034 & OD1 & OD1 genus incertae sedis \\
\hline & Mild & 0.804 & 0.022 & Proteobacteria & $\begin{array}{l}\text { Alphaproteobacteria } \\
\text { unclassified }\end{array}$ \\
\hline & Mild & 0.796 & 0.04 & Bacteroidetes & Chitinophagaceae unclassified \\
\hline & Mild & 0.794 & 0.02 & Proteobacteria & Rhodobacter \\
\hline & Mild & 0.751 & 0.05 & Proteobacteria & $\begin{array}{l}\text { Gammaproteobacteria } \\
\text { unclassified }\end{array}$ \\
\hline & Moderate & 0.761 & 0.027 & Proteobacteria & Burkholderiales unclassified \\
\hline \multicolumn{6}{|c|}{$\begin{array}{l}\text { Bd Infection Status } \\
\text { (AR Only) }\end{array}$} \\
\hline & $B d-$ & 0.888 & 0.05 & Proteobacteria & Aquabacterium \\
\hline & $\mathrm{Bd}+$ & 0.902 & 0.029 & Proteobacteria & Novosphingobium \\
\hline & $B d+$ & 0.881 & 0.035 & Bacteroidetes & Sediminibacterium \\
\hline & $B d+$ & 0.878 & 0.032 & Proteobacteria & $\begin{array}{l}\text { Sphingomonadaceae } \\
\text { unclassified }\end{array}$ \\
\hline & $B d+$ & 0.816 & 0.012 & Chloroflexi & Chloroflexi unclassified \\
\hline & $B d+$ & 0.707 & 0.048 & Proteobacteria & Neisseriaceae unclassified \\
\hline
\end{tabular}

A total of 136 phylotypes were shared between lesion severity groups within AR, with 17 unique to animals with mild lesions, 50 to animals with moderate lesions, and 48 to animals with severe lesions. Increased abundance in Firmicutes and Synergistetes was correlated with moderate and severe lesion groups for AR hellbenders (Fig. 7, Table 2). Indicator species analysis identified 27 phylotypes representative of individuals with mild toe lesions, only one for those with moderate lesions, and none for those with severe lesions (Table 3). Top indicator phylotypes (IndVal Index stat >0.9) for mild lesions were species in the genera Opitutus, Emticicia, Ferruginibacter, Zavarzinella, and an unclassified of order Myxococcales. Five of the 27 indicator phylotypes for mild lesions within AR were also indicators for ETN, including three out of the five top mild lesion indicator taxa Opitutus, Ferruginibacter, and an unclassified order Myxococcales. The only indicator species for animals with moderate lesions was an unclassified phylotype in the order Burkholderiales.

Although bacterial alpha diversity and assemblage structure was not significant between $B d$ positive and negative individuals, indicator species analysis identified one phylotype (Aquabacterium) indicative of $B d$ negative skin 
assemblages and five for $B d$ positive skin (Table 3). For life stage analysis within ETN, we identified a total of 10 phylotypes as indicative of adult skin, and 10 of juvenile skin, none with a IndVal Index stat >0.9 (Table 3)

\section{Discussion}

Our results showed differences in dorsal skin bacterial assemblage structure and diversity between two subspecies of hellbenders from populations not previously sampled. Furthermore, we showed that variation in these communities could be explained by subspecies, severity of chronic lesions in the feet, and partially by life stage. Bd infection status and adult size class (mass) were not correlated with dorsal skin bacterial assemblages. Several factors including both environment and host health may be contributing to these differences. Host populations are separate subspecies from different ecoregions. Furthermore, the ETN eastern hellbender population we sampled is considered healthy and stable and surrounded by forested land (USFWS 2018; Da Silva Neto et al. 2019) whereas the AR Ozark population is in danger of extirpation with little surrounding forest buffer (USFWS 2018).

Distinction between ETN and AR dorsal skin microbial assemblage was greatest via Sorenson Index for average assemblage structure and via lower Hill numbers for alpha diversity. This highlights that subspecies differences were driven by presence of relatively rarer phylotypes. Similar to other studies, we determined that skin microbial assemblage structure of hellbenders differed by subspecies (Hernández-Gómez et al. 2017b, 2018). However, in contrast to those previous studies, we showed eastern hellbenders to have greater richness. We sampled Ozark hellbenders from AR and eastern hellbenders from ETN, whereas previous work focused on populations of both subspecies within Missouri (MO). This is an important distinction for eastern hellbender sampling. While Ozark hellbenders have a small contiguous range within the Ozark highlands of AR and MO only, eastern hellbenders span a large geographic range with eastern $\mathrm{MO}$ populations disjunct from all other eastern hellbenders and phylogenetically distinct from ETN (Unger et al. 2013; Hime 2017). Disparate results are further confounded by differential population health within the eastern hellbender subspecies. Eastern hellbenders of MO have been classified as an endangered population alongside the Ozark subspecies whereas the rest of the eastern hellbenders remain unlisted (USFWS 2018).

Interestingly, we found a trend of decreased alpha diversity with increased lesion severity with the apparently unhealthy Ozark hellbender population of AR. This, along with subspecies differences, may suggest that skin bacterial diversity is negatively associated with hellbender skin health. This is in contrast to Hernández-Gómez et al. (2017b) who found toe lesions to have richer assemblages than apparently healthy dorsal skin within a given individual. However, our study differed in that we compared dorsum between individuals of varying toe lesion severity, as opposed to direct comparison between lesioned and lesion-free skin within an individual. Furthermore, along with decreased diversity, we showed skin microbiomes of AR hellbenders, particularly those with severe lesions, had increased beta dispersion. This suggests severe lesions may be associated with uneven, random, and potentially dysbiotic, skin bacterial communities. In contrast to subspecies comparisons, dorsal skin bacterial assemblage differences between AR individuals of varying toe lesion severity were driven by relative abundance of more common phylotypes. A similar relationship was observed between dorsum and toe lesions in Ozark hellbenders of MO in that differences were driven by changes in relative abundance of shared OTUs (Hernández-Gómez et al. 2017b).

These distal limb lesions are progressive and can take several years to increase in severity (Hardman et al. 2020a), and the same factors affecting toe lesions may also be affecting dorsal skin communities. Changes we observed between AR hellbenders of varying toe lesion severity could represent a decreased ability to maintain an optimal microbial community as disease progresses in an individual. Another explanation could be that local microbial changes due to chronic lesions can eventually generate changes over the entire skin surface as has been seen around

Page $15 / 28$ 
chronic ulceration due to leishmaniasis in humans (Gimblet et al. 2017). Alternatively, shifts in microbial communities could be the main mechanism by which lesions manifest, and lesion severity represent a sequela of this dysbiosis that manifests specifically in an area of increased use (e.g. toes). The mechanism by which these patterns may arise is still speculative and it is possible these trends are not causal or are potentially spurious from limited sample size within AR ( $n=15)$. More intensive within-river surveys alongside more detailed measurements of health (i.e., blood and immunological parameters, body condition score, and detailed lesion scoring) will be needed to better understand within watershed variation and drivers from host health and environment on changes in skin bacterial community parameters.

Unexpectedly, infection status of $B d$ had no effect on beta or alpha diversity. This was an unexpected result based on other studies of $B d$ infection being associated with skin microbial community shifts in amphibians (Jani and Briggs 2014; Muletz-Wolz et al. 2019). Wild hellbenders appear to be tolerant to $B d$ infection and there are no confirmed reports of chytridiomycosis in a wild hellbender despite the impressive amount of $B d$ surveillance studies with often 20-30\% infection prevalence (Gonynor et al. 2011; Souza et al. 2012; Bales et al. 2015; Seeley et al. 2016; Hardman et al. 2020b). However, $B d$ may be an important secondary invader during instances of decreased skin immunity as chytridiomycosis is reported in captive and stressful conditions (Dean et al. 2016; Dusick et al. 2017), and Bd infection rates are positively associated with higher lesion scores in wild AR populations (Hardman et al. 2020a). Still, data from this study suggest that even if $B d$ affects AR hellbender skin health, it is not in association with changes in the skin bacterial community.

Life stage also had minimal effect on the bacterial skin community, at least within the ETN population for which we were able to sample juveniles. Similar to subspecies differences, community membership but not relative abundance, was predictive of hellbender life stage. Our study was limited in the fact that only two juveniles were still gilled larvae (pre-metamorphosis). Evaluation of larval and post-metamorphic juvenile substages within a single site may reveal significant microbiome shifts after metamorphosis we were unable to evaluate.

We found commonalities in indicator taxa that overlapped with those found in previous hellbender studies. The top indicator phylotypes for AR (genera Limnohabitans and Acinetobacter) belonged to families Comamonadaceae and Moralleaceae, respectively. These taxa were previously identified with increased abundance in toe lesions compared to dorsal skin in Ozark hellbenders of MO (Hernández-Gómez et al. 2017b), and further characterized as significantly more abundant in Ozark vs eastern hellbender skin of MO (Hernández-Gómez et al. 2018). The genus Acinetobacter is of particular interest as an indicator taxon because this genus has been previously cultured from Ozark hellbender toe lesions (Nickerson et al. 2011) and is also highly correlated with differential amphibian host responses to $B d$ infection in both laboratory and field experiments (Bates et al. 2018). Similarly, the only indicator taxon for moderate lesions was an unclassified Burkholderiales, and an OTU in the Burkholderiales was recently shown to increase in abundance on skin of Sierra Nevada yellow-legged frogs, Rana sierrae, experimentally infected with $B d$ (Ellison et al. 2019).

Some phylotypes may associate with healthy hellbender skin. Several of the indicator taxa that distinguished dorsal skin assemblages of AR animals with mild versus moderate or severe toe lesions were the same that distinguished ETN from AR. One particular (genus Ferruginibacter) has also been identified as a core taxon on alpine newt (Ichthyosaura alpestris) skin (Bletz et al. 2017b).

No indicator phylotype for ETN eastern hellbenders from this study overlapped with taxa previously identified as significantly associated with eastern hellbender skin. As mentioned above, our study evaluated eastern hellbenders from ETN whereas the previous studies comparing hellbender subspecies evaluated those from MO. Populations

Page 16/28 
from ETN and MO vary greatly in phylogeny, ecoregion, and population health. Conversely, AR Ozark hellbenders are phylogenetically very similar to MO Ozark hellbenders and individuals sampled from our study were from one of the same watersheds sampled in these previous studies. It is no surprise then that our indicator species analysis revealed similar results for Ozark hellbenders yet disparate results for eastern hellbenders. The importance of specific taxa of bacteria and their function within a community context remains an understudied area in amphibian microbiome research. This study contributes to our understanding of the microbial assemblages of hellbenders and their correlation with skin health.

\section{Conclusions}

Our study demonstrated that bacterial assemblages on hellbender dorsal skin are not only associated with subspecies but could be shaped by skin health. Regardless of the immediate mechanism driving hellbender skin microbial communities, continued evaluation of their structure and diversity, when added to traditional amphibian disease surveillance, may provide a more comprehensive assessment of wild population health. We found a correlation between decreased dorsal skin bacterial assemblage similarity and richness within Ozark hellbenders compared to eastern hellbenders. This trend was further explained by severity of toe lesions in Ozark hellbenders. This pattern was not affected by presence of $B d$ on the skin and was likely related to other factors linked with chronic changes in the host and/or aquatic environment. These salamanders are long lived, river-dependent organisms with documented population declines associated with habitat changes, especially sedimentation. The skin environment is constantly exposed overtime to both this altered lotic environment as well as its secondary effects on host skin maintenance. Intensive sampling will be required in both healthy and unhealthy populations of both subspecies to disentangle factors affecting these important skin microbial communities, and to ultimately inform management to maintain and promote future healthy wild hellbenders.

\section{Methods}

We sampled hellbenders from 2014-2015 from mid-July to mid-August in two rivers, one from the Blue Ridge ecoregion of eastern Tennessee (ETN), the other in the Ozark Highlands of Arkansas (AR) (Fig. 1). We sampled in ETN for the eastern hellbender (C. a. alleganiensis) and in AR for the Ozark hellbender (C. a. bishopi). All sampling in Tennessee with approval from Tennessee Wildlife Resources Agency (TWRA) (Permit \# 1877 (RHH)) and in AR under observation of the USFWS permit TE66039A-0 issued to KJI and Arkansas Game and Fish Commission. In TN, and shallower water in AR, we used standard snorkeling techniques to locate individuals. In AR, we also sampled hellbenders via artificial nest boxes (Briggler and Ackerson 2012) and sampled deep water habitats (up to 4 meters) using a hookah dive system (i.e. gasoline powered air compressor with tethered air supply lines to dive regulators). We captured any animals encountered under cover objects and nest boxes and placed them in a clean, soft cotton or mesh bag. We kept bags submerged in the river before and after animals were processed. (University of Tennessee IACUC protocol \# 2481 - 0916). We changed dive gloves between animal captures to reduce pathogen transfer and contamination of samples. Skin microbiome samples were collected from a subset of hellbenders surveyed for disease and amphibian pathogen prevalence (see Hardman et al. 2020 a,b). We placed each individual in a new, cleaned plastic tub and rinsed the dorsum with approximately $200 \mathrm{~mL}$ sterile distilled water. We immediately rubbed a sterile cotton swab (Fisherbrand product\# 23400111) over the dorsal skin surface for 30 seconds and placed it in a sterile $1.5 \mathrm{~mL}$ tube on dry ice for transport to a $-80^{\circ} \mathrm{C}$ freezer. For individuals in AR only, we also obtained a swab for $B d$ load analysis for a concurrent pathogen prevalence study (see Hardman et al. 2020b), as well as, lesion severity score (scored from 0-7) for toe lesions of unknown etiology (Hardman et al. 2020a). All individuals sampled from AR for the microbiome analysis had toe lesions, but because of low sample size within AR, and subsequent inability to

Page 17/28 
compare across all 8 lesion scores, we binned scores of 0-7 into three biologically relevant severity levels of mild (score 0-2; toe swelling only), moderate (3-4; toe swelling and some shortened) and severe (5-7; most toes shortened and/or missing).

\section{Amplicon Library Sequence Preparation}

We extracted DNA from skin swabs using ZR Fungal/ Bacterial DNA Miniprep kits (Zymo Research Corporation, Irvine, CA, USA) and sent extracted DNA to Hudson Alpha (Huntsville, AL) for 16S rDNA amplicon sequencing on the Illumina MiSEQ platform ( v2 2 × 250) of the V3-V4 region of 350 bp length. Bioinformatic preparation of the resulting sequence data was performed in the program MOTHUR, following the MiSeq protocol (Schloss et al. 2009) with minor modifications including the creation of an oligo file of primer sequences to recognize the target V3-V4 region from primer trimming after making contigs. We used a phylotype based approach to assign phylotypes to the rank of genus based on the Silva v128 reference database (Quast et al. 2013; Yilmaz et al. 2014).

\section{Statistical Analysis}

We performed all subsequent cleanup and analyses using RStudio v1.2.5019 (R Studio Team 2016). We removed rare phylotypes with less than five total sequence reads or occurring in less than three samples. We also removed samples with less than 4000 sequence reads based on rarefaction curves performed in package Vegan (Oksanen et al. 2015). To compare alpha diversity among samples we used packages Vegetarian (Charney and Record 2012) and Vegan to calculate an averaged 1000 iteration bootstrapped value generated from 1000 sequence subsamples for the following Hill numbers: relativized richness $(q=0)$, exponential of Shannon's index $(q=1)$ and the inverse Simpson's index $(q=2)$. We used Hill numbers as they provide better evaluation of changes in both richness and evenness (Chao et al. 2014). We performed the same bootstrap technique to calculate beta diversity distance matrices of the Sorenson Index and Morisita-Horn to evaluate structural changes based on phylotype presence/absence and relative abundance, respectively. We performed all statistical comparisons of both beta and alpha diversity in package Vegan. For alpha diversity analysis we normalized each response variable (either natural log or cubed transformation) and performed an analysis of variance (ANOVA) across all samples to test for variation in alpha diversity by subspecies and mass. We performed a second ANOVA from AR samples only to test for variation in alpha diversity by $B d$ infection status and lesion severity within this population. A final ANOVA was performed to evaluate variation in alpha diversity that correlated with life stage effects (juvenile vs adult) within ETN only where both adults and juveniles were encountered. We used PERMAVOVA (adonis - 999 permutations) to test for differences in average assemblage structure using Sorenson Index and Morisita-Horn distances and previously mentioned categorical predictors mentioned for the alpha diversity analysis. The only exception was that mass was binned into natural breaks of adult size class of small (150-299g), medium (300-449), and large (450+) adults. We also assessed beta diversity as a multivariate dispersion (community consistency among samples) by calculating the multidimensional area of minimum convex polygons fit to clusters using the 'betadisp' function within vegan based on distances created from principal coordinates analysis (PCOA) from previously calculated Sorenson and Morisita-Horn indices. For visualization of alpha diversity differences, we created boxplots for each Hill number for each parameter using package ggplot2 (Wickham 2016). For visualization of beta diversity between these same groups we created nonmetric multidimensional scaling (NMDS) plots based on distance matrices via packages Vegan and ggplot2.

\section{Bacterial Taxonomic Analysis}

We calculated and graphed phylotype abundance by bacterial phylum for a single subsample of 1000 sequences for each sample in package phyloseq (McMurdie and Holmes 2013). Finally, we identified significant indicator phylotypes for each comparison group via the indicspecies package (De Caceres and Legendre 2009) using the 
'multipatt' function with 'IndVal' setting, considering top indicator phylotypes those with a p value $<0.05$ and IndVal Index stat $>0.9$.

\section{Abbreviations}

AR

ETN

OTU

MO

ANOVA

NMDS

\section{Declarations}

\section{Ethics approval and consent to participate}

This study was approved by the University of Tennessee IACUC Committee. Protocol \#2481

\section{Consent for publication}

Not applicable

\section{Availability of data and materials}

The datasets supporting the conclusions of this article are available in the NCBI SRA repository, [PRJNA755662, http://www.ncbi.nlm.nih.gov/bioproject/755662] and included within the article additional files (for sequence and SRA metadata and MOTHUR and R code, respectively).

\section{Competing interests}

The authors declare that they have no competing interests

\section{Funding}

Funding was provided by the Arkansas Game and Fish Commission

\section{Authors' contributions}

$\mathrm{RHH}$ contributed to project inception, field and laboratory work, data analysis and manuscript writing. GL contributed to project inception, sample preparation, data analysis, and manuscript editing. BF contributed to data analysis, and manuscript editing. KJI contributed to project inception, acquisition of funds and field collection. WBS, JGD, and MJF contributed to field collection, manuscript editing, and sample transport. DMW contributed to data analysis. DLM contributed to project inception, acquisition of funds, project oversight, and manuscript editing.

\section{Acknowledgements}


We would like to thank the staff of the Arkansas Game and Fish Commission and Arkansas office of the USFWS, especially Chris Davidson and Erin Knoll for help with Arkansas field work. Thank you to Jessica Braunstein and Ruth Freemon for help with Tennessee field work. Thanks to Davis Carter for help with laboratory preparation.

\section{Authors' information \\ Endnotes}

\section{References}

1. Amato KR, Teoman CJ, Kent A, Righini N, Carbornero F, Estrada A, Gaskins HR, Stumpf RM, Yildirim S, Torralba M, Gillis M, Wilson BA, Nelson KE, White BA, Leigh SR. Habitat degradation impacts black howler monkey (Alouatta pigra) gastrointestinal microbiomes. The ISME Journal 2013;7:1344-53.

2. Arnolds KL, Lozupone CA. Striking a balance with help from our little friends-how the gut microbiota contributes to immune homeostasis. Yale J Biol Med. 2016;89:389-95.

3. Bales EK, Hyman OJ, Loudon AH, Harris RN, Lipps G, Chapman E, Roblee K, Kleopfer JD, Terrell KA. Pathogenic chytrid fungus Batrachochytrium dendrobatidis, but not $B$. salamandrivorans, detected on eastern hellbenders. PLoS One 2015;10:e0116405.

4. Bates KA, Clare FC, O’Hanlon S, Bosch J, Brookes L, Hopkins K, McLaughlin EJ, Daniel O, Garner TWJ, Fisher MC. Amphibian chytridiomycosis outbreak dynamics are linked with host skin bacterial community structure. Nat Comm. 2018;9:693.

5. Becker $\mathrm{MH}$, Harris RN. Cutaneous bacteria of the redback salamander prevent morbidity associated with a lethal disease. PLoS ONE 2010;5:e10957.

6. Bernardo-Cravo AP, Schmeller DS, Chatzinotas A,Vredenburg VT, Adeline L. Environmental factors and host microbiomes shape host-pathogen dynamics. Trends in Parasitology 2020;36:616-33.

7. Bletz MC, Myers J, Woodhams DC, Rabemananjara FCE, Rakotonirina A, Weldon C, Edmonds D, Vences M, Harris RN. Estimating herd immunity to amphibian chytridiomycosis in Madagascar based on the defensive function of amphibian skin bacteria. Frontiers in Microbiology 2017a;8:1751.

8. Bletz MC, Perl RGB, Vences M. Skin microbiota differs drastically between co-occurring frogs and newts. R. Soc. open sci. 2017b;4:170107.

9. Chao A, Gotelli NJ, Hsieh TC, Sander EL, Ma KH, Colwell RK, Ellison AM. Rarefaction and extrapolation with Hill numbers: a framework for sampling and estimation in species diversity studies. Ecological Monographs 2014;84:45-67.

10. Charney N, Record S. Vegetarian: Jost Diversity Measures for Community Data. R package version 1.2. 2018. https://CRAN.R-project.org/package=vegetarian.

11. Da Silva Neto JG, Sutton WB, Freake MJ. Life-stage differences in microhabitat use by hellbenders (Cryptobranchus alleganiensis). Herpetologica 2019;75:21-9.

12. Dean N, Ossiboff R, Bunting E, Schuler K, Rothrock A, Roblee K. The eastern hellbender and Batrachochytrium dendrobatidis (Bd) in western New York. Presented at the 65th Annual International Conference of the Wildlife Disease Association, Cortland, NY. 2016.

13. De Caceres M, Legendre P. Associations between species and groups of sites: indices and statistical inference. Ecology 2009;90:3.

14. de Vos WM, de Vos EAJ. Role of intestinal microbiome in health and disease: from correlation to causation. Nutrition Reviews 2012;70:545-56. 
15. Dusick A, Flatland B, Craig L, Ferguson S. What is your diagnosis? Skin scraping from a hellbender. Veterinary Clinical Pathology 2017;46:183-4.

16. Ellison S, Knapp RA, Sparagon W, Swei A, Vrendenburg VT. Reduced skin bacterial diversity correlates with increased pathogen infection intensity in an endangered amphibian host. Molecular Ecology. 2019;28:127-40.

17. Fitzpatrick BM, Allison AM. Similarity and differentiation between bacteria associated with skin of salamanders (Plethodon jordani) and free-living assemblages. FEMS Microbiology Ecology 2014;88:482-94.

18. Gimblet C, Meisel JS, Loesche MA, Cole SD, Horwinski J, Novais FO, Misic AM, Bradley CW, Beiting DP, Rankin SC, Carvalho LP, Carvalho EM, Scott P, Grice EA. Cutaneous leishmaniasis induces a transmissible dysbiotic skin microbiota that promotes skin inflammation. Cell Host Microbe. 2017;22:13-24.e4.

19. Glasl B, Herndl GJ, Frade PR. The microbiome of coral surface mucus has a key role in mediating holobiont health and survival upon disturbance. The ISME Journal 2016;10:2280-92.

20. Gonynor JL, Yabsley MJ, Jensen JB. A preliminary survey of Batrachochytrium dendrobatidis exposure in hellbenders from a stream in Georgia, USA. Herpetological Review 2011;42:58-9.

21. Grice EA, Segre JA. The skin microbiome. Nature Reviews Microbiology 2011;9:626.

22. Grice EA, Segre JA. Interaction of the microbiome with the innate immune response in chronic wounds. Adv Exp Med Biol. 2012;946:55-68.

23. Hardman RH, Irwin KJ, Sutton WB, Miller DL. Evaluation of severity and factors contributing to foot lesions in endangered Ozark Hellbenders, Cryptobranchus alleganiensis bishopi. Frontiers in Veterinary Science 2020a;7:110.

24. Hardman RH, Sutton WB, Irwin KJ, McGinnity D, Reinsch SD, Freake M., Colclough P, Miller BT, Da Silva Neto JG, Souza MJ, Fitzpatrick B, Miller DL. 2020b. Geographic and individual determinants of important amphibian pathogens in hellbenders (Cryptobranchus alleganiensis) in Tennessee and Arkansas, USA. Journal of Wildlife Diseases 2020b;56:803-14.

25. Hernández-Gómez O, Hoverman JT, Williams RN. Cutaneous microbial community variation across populations of Eastern hellbenders (Cryptobranchus alleganiensis alleganiensis). Frontiers in Microbiology 2017a;8:1-16.

26. Hernández-Gómez O, Kimble SJA, Briggler JT, Williams RT. Characterization of the cutaneous bacterial communities of two giant salamander subspecies. Microbial Ecology 2017b;73:445-54.

27. Hernández-Gómez O, Briggler JT, Williams RN. Influence of immunogenetics, sex and body condition on the cutaneous microbial communities of two giant salamanders. Molecular Ecology 2018;27:1915-29.

28. Hime PM. Genomic perspectives on amphibian evolution across multiple phylogenetic scales. https://uknowledge.uky.edu/biology_etds/45 (2017). Accessed 11 Nov 2018.

29. Hopkins WA, DuRant SA. Innate immunity and stress physiology of eastern hellbenders (Cryptobranchus alleganiensis) from two stream reaches with differing habitat quality. General and Comparative Endocrinology 2011;174:107-15.

30. Humphries WJ, Pauley TK. Life history of the hellbender, Cryptobranchus alleganiensis, in a West Virginia stream. American Midland Naturalist 2005;154:135-42.

31. Ilseung C, Blaser MJ. The human microbiome: at the interface of health and disease. Nature Review Genetics 2012;13:260-70.

32. Jani AJ, Briggs CJ. The pathogen Batrachochytrium dendrobatidis disturbs the frog skin microbiome during a natural epidemic and experimental infection. PNAS 2014;111:E5049-E5058

33. Jani AJ, Briggs CJ. Host and aquatic environment shape the amphibian skin microbiome but effects on downstream resistance to the pathogen Batrachochytrium dendrobatidis are variable. Frontiers in Microbiology

Page $21 / 28$ 
2018;9:487.

34. Jani AJ. Amphibian microbiome linked to climate. Nature Ecology and Evolution 2019;3:332-333.

35. Jiménez RR, Sommer S. The amphibian microbiome: natural range of variation, pathogenic dysbiosis, and role in conservation. Biodiversity and Conservation 2017;26:763.

36. Kang D, Adams JB, Gregory AC, Borody T, Chittick L, Fasano A, Khoruts A, Geis E, Maldonado J McDonoughMeans S, Pollard EL, Roux S, Sadowsky MJ, Schwarzberg Lipson K, Sullivan MB, Caporaso JG, Krajmalnik-Brown R. Microbiota transfer therapy alters gut ecosystem and improves gastrointestinal and autism symptoms: an open-label study. 2017;Microbiome 5:10.

37. Krediet CJ, Ritchie KB, Paul VJ, Teplitski M. Coral-associated micro-organisms and their roles in promoting coral health and thwarting diseases. Proceedings of the Royal Society B: Biological Sciences 2013;280:20122328.

38. Kueneman JG, Bletz MC, McKenzie VJ, Becker CG, Joseph MB, Abarca JG, Archer H, Arellano AL, Bataille A, Becker M, Belden LK, Crottini A, Geffers R, Haddad CFB, Harris RN, Holden WM, Hughey M, Jarek M, Kearns PJ, Kerby JL, Kielgast J, Kurabayashi A, Longo AV, Loudon A, Medina D, Nuñez JJ, Bina Perl RG, Pinto-Tomás A, Rabemananjara FCE, Rebollar EA, Rodríguez A, Rollins-Smith L, Stevenson R, Tebbe CC, Asensio GV, Waldman B, Walke JB, Whitfield SM, Zamudio KR, Chaves IZ, Woodhams DC, Vences M. Community richness of amphibian skin bacteria correlates with bioclimate at the global scale. Nature Ecology and Evolution 2019;3:381-9.

39. Küng D, Bigler L, Davis LR, Gratwicke B, Griffith E, Woodhams DC. Stability of microbiota facilitated by host immune regulation: informing probiotic strategies to manage amphibian disease. PLoS ONE 2014;9:e87101.

40. McKenzie VJ, Bowers RM, Fierer N, Knight R, Lauber CL. Co-habiting amphibian species harbor unique skin bacterial communities in wild populations. The ISME Journal 2011;6:588-96.

41. McMurdie PJ, Holmes S. phyloseq: An R package for reproducible interactive analysis and graphics of microbiome census data. PLoS ONE 2013;8:e61217.

42. Muletz-Wolz CR, Fleischer RC, Lips KR. Fungal disease and temperature alter skin microbiome structure in an experimental salamander system. Mol Ecol. 2019;28:2917-2931.

43. Nickerson CA, Ott CM, Castro SL, Garcia VM, Molina TC, Briggler JT, Pitt AL, Tavano JJ, Byram JK, Barrila J, Nickerson MA. Evaluation of microorganisms cultured from injured and repressed tissue regeneration sites in endangered giant aquatic Ozark hellbender salamanders. PLoS ONE 2011;6:e28906.

44. Oksanen J, Blanchet FG, Friendly M, Kindt R, Legendre P, McGlinn D, and Minchin PR, O'Hara RB, Simpson GL, Solymos P, Stevens MHH, Szoecs E, Wagner H. vegan: Community Ecology Package, R package version 2.5-4. 2019. https://CRAN.R-project.org/package=vegan.

45. Pang M, Zhu M, Lei X, Xu P, Cheng B. Microbiome imbalances: an overlooked potential mechanism in chronic nonhealing wounds. The International Journal of Lower Extremity Wounds 2019;18:31-41.

46. Pugh MW, Hutchins M, Madritch M, Slefferman L, Gangloff MM. Land-use and local physical and chemical habitat parameters predict site occupancy by hellbender salamanders. Hydrobiologia 2015;770:105-16.

47. Quast C, Pruesse E, Yilmaz P, Gerken J, Schweer T, Yarza P, Peplies J, Glöckner FO The SILVA ribosomal RNA gene database project: improved data processing and web-based tools. Nucl. Acids Res. 2013;41:D590-96.

48. Rodriguez Hoffman A, Proctor LM, Surette MG, Suchodolski JS. The microbiome: the trillions of microorganisms that maintain health and cause disease in human and companion animals. Veterinary Pathology 2015;53:10-21.

49. RStudio Team RStudio: Integrated Development Environment for R. RStudio, Inc. 2016. http://www.rstudio.com.

50. Scales BS, Huffnagle GB. The microbiome in wound repair and tissue fibrosis. The Journal of Pathology 2012;229:323-31.

Page 22/28 
51. Scheele BC, Pasmans F, Skerratt LF, Berger L, Martel A, Beukem A, Acevedo AA, Burrowes PA, Carvalho T, Catenazzi A, De la Riva I, Fisher MC, Flechas SV, Foster CN, Frias-Alvarez P, Garner TWJ, Gratwicke B, Guayasamin JM, Hirschfeld M, Kolby JE, Kosch TA, La Marca E, Lindenmayer DB, Lips KR, Longo AV, Maneyro R, McDonald CA, Mendelson III J, Palacios-Rodriguez P, Parra-Olea G, Richards-Zawacki CL, Rodel M-O, Rovito SM, Soto-Azat C, Toledo LF, Voyles J, Weldon C, Whitfield SM, Wilkinson M, Zamudio KR, Canessa S. Amphibian fungal panzootic causes catastrophic and ongoing loss of biodiversity. Science 2019;363:1459-63.

52. Schloss PD, Westcott SL, Ryabin T, Hall JR, Hartmann M, Hollister EB, Lesniewski RA, Oakley BB, Parks DH, Robinson CJ, Sahl JW, Stres B, Thallinger GG, Van Horn DJ, Weber CF. Introducing mothur: Open-source, platform-independent, community-supported software for describing and comparing microbial communities. Appl Environ Microbiol, 2009;75:7537-41.

53. Seeley KE, D'Angelo M, Gowins C, Greathouse J. Prevalence of Batrachochytrium dendrobatidis in eastern hellbender (Cryptobranchus alleganiensis) populations in West Virginia, USA. Journal of Wildlife Diseases 2016;52:391-4.

54. Shoemaker VH, Nagy K. Osmoregulation in amphibians and reptiles. Annual Review of Physiology 1977;39:44971.

55. Souza MJ, Gray MJ, Colclough P, Miller DL. Prevalence of infection by Batrachochytrium dendrobatidis and ranavirus in eastern hellbenders (Cryptobranchus alleganiensis alleganiensis) in eastern Tennessee. Journal Wildlife Diseases 2012;48:560-6.

56. Shreiner AB, Kao JY, Young VB. The gut microbiome in health and in disease. Current Opinions in Gastroenterology 2015;31:69-75.

57. Thio HB. The microbiome in psoriasis and psoriatic arthritis: The skin perspective. Journal of Rheumatology 2018;94 Suppl 30-31.

58. Unger SD, Rhodes OE Jr, Sutton TM, Williams RN. Population genetics of the eastern hellbender (Cryptobranchus alleganiensis alleganiensis) across multiple spatial scales. PLoS ONE 2013;8:e74180.

59. USFWS. Endangered and Threatened Wildlife and Plants; Endangered Status for the Ozark hellbender Salamander. 50 CFR Part 23. Federal Register 2011;76:61956-78.

60. USFWS. Species status assessment report for the Eastern hellbender (Cryptobranchus alleganiensis alleganiensis). https://www.fws.gov/midwest/endangered/amphibians/pdf/Eastern_Hellbender_SSA_Report.pdf (2018). Accessed 17 Jan 2019.

61. Wickham H. ggplot2: Elegant Graphics for Data Analysis, New York: Springer-Verlag; 2016.

62. Yilmaz P, Parfrey LW, Yarza P, Gerken J, Pruesse E, Quast C, Schweer T, Peplies J, Ludwig W, Glöckner FO. The SILVA and "All-species Living Tree Project (LTP)" taxonomic frameworks. Nucl. Acids Res. 2014;42:D643-8.

\section{Figures}




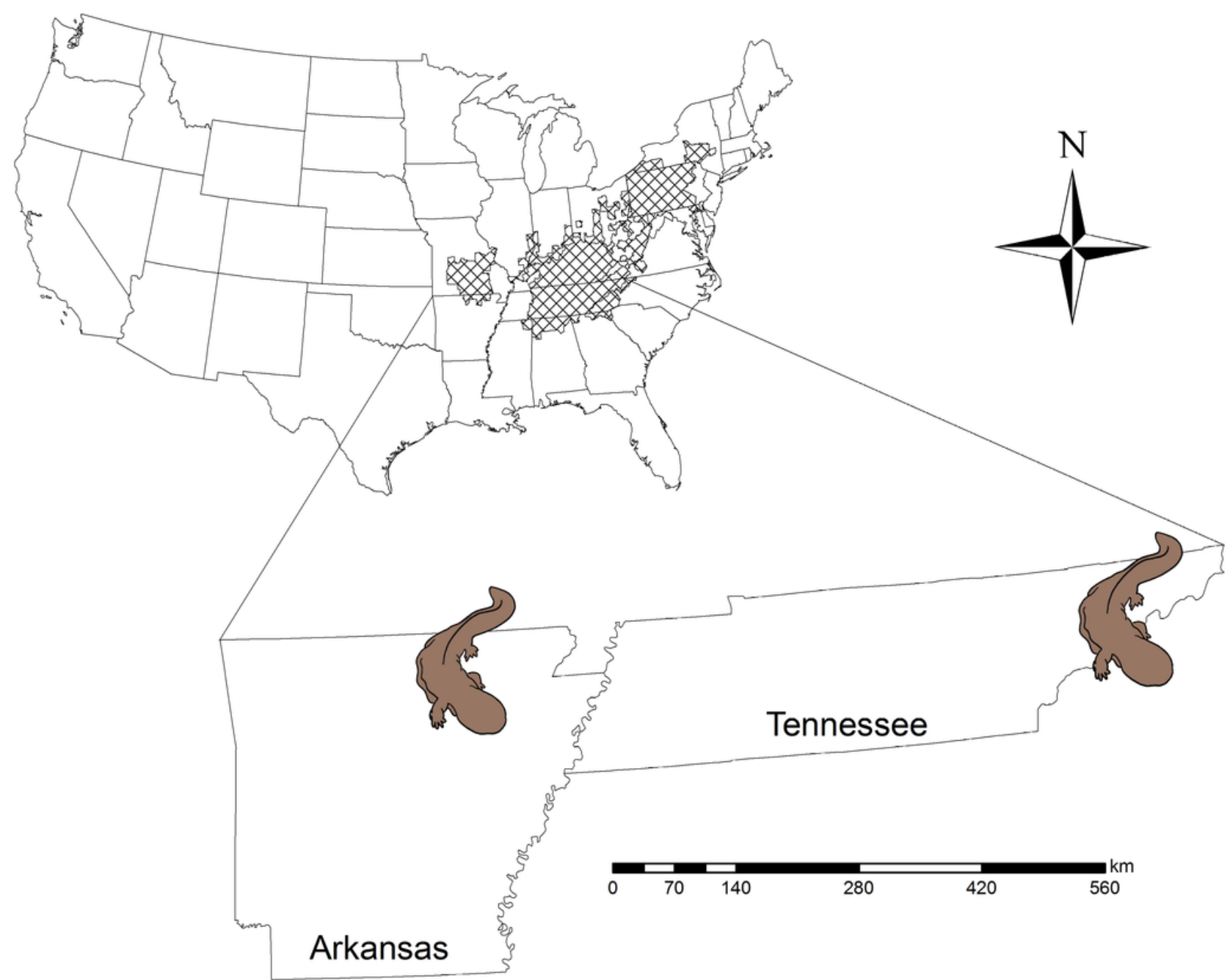

Figure 1

Map of United States displaying historical range of hellbenders (Cryptobranchus alleganiensis) with cross hatching and zoomed insert displaying relative location of field sampling denoted by hellbender icons in Arkansas (AR) and eastern Tennessee (ETN). Ozark hellbenders (C. a. bishop) were sampled in AR along with collection Batrachochytrium dendrobatidis (Bd) skin swabs and observations of toe lesions. Eastern hellbenders (C. a. alleganiensis) were sampled in ETN where both adult and juveniles were encountered. 

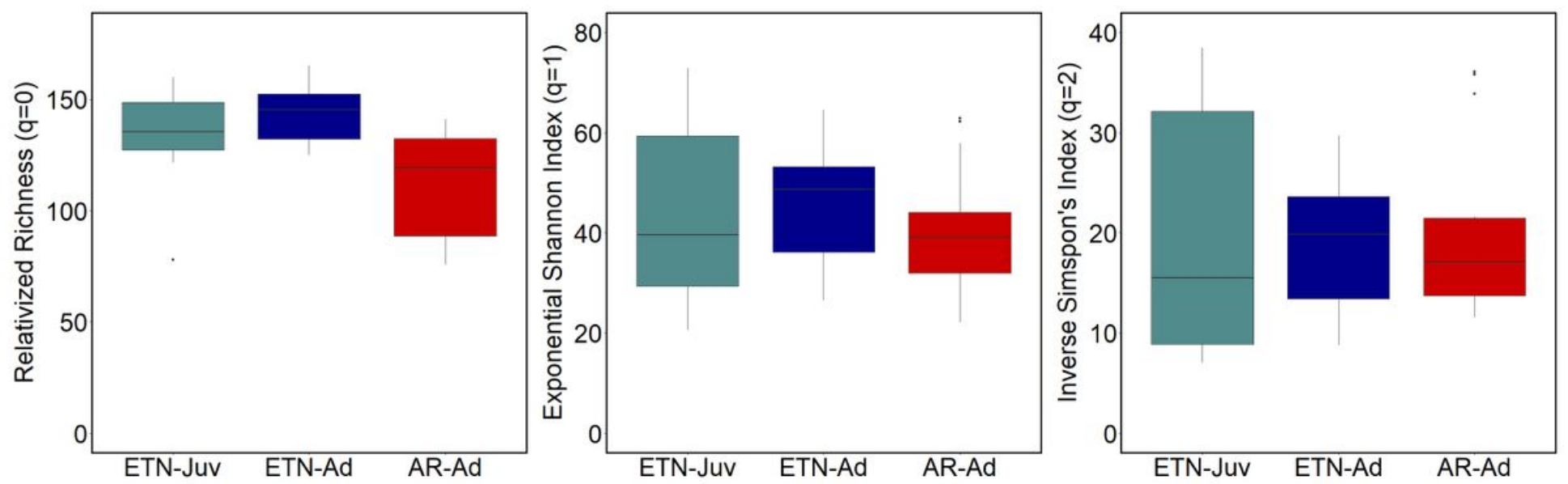

\section{Figure 2}

Alpha diversity comparison of bacterial 16S assemblages from dorsal skin swabs of hellbenders (Cryptobranchus alleganiensis) using Hill numbers from order $q=0$ (relativized richness), $q=1$ (exponential of Shannon Index) and $q=2$ (inverse Simpson's Index). Comparison between eastern hellbenders (C. a. alleganiensis) of eastern Tennessee (ETN) and Ozark hellbenders (C. a. bishopi) of Arkansas (AR). ETN hellbenders are further grouped into juveniles and adults.
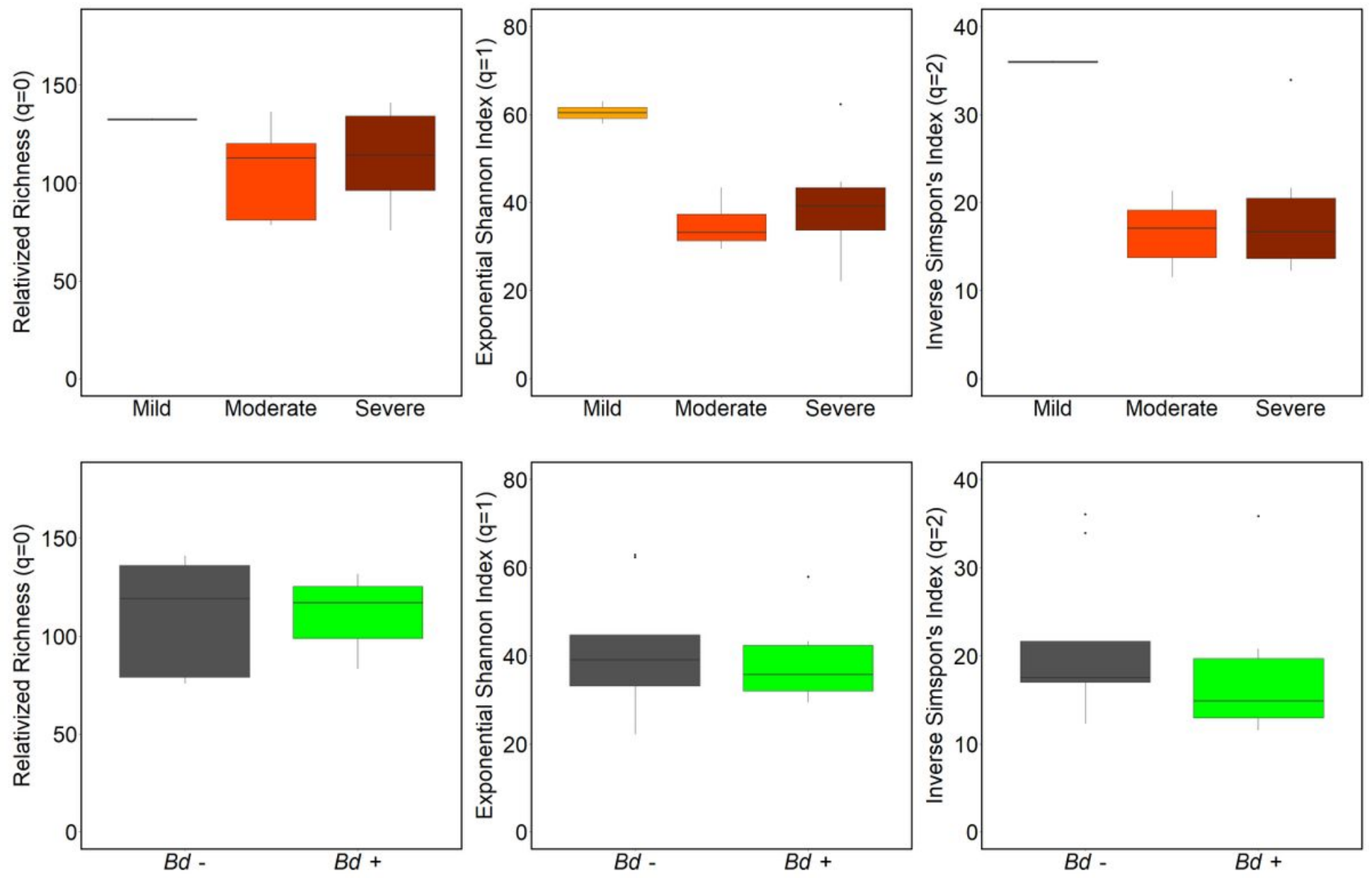

Figure 3 
Alpha diversity comparison of bacterial 16S assemblages from dorsal skin swabs of Ozark hellbenders (Cryptobranchus alleganiensis bishopi) using Hill numbers from order $q=0$ (relativized richness), $q=1$ (exponential of Shannon Index) and q=2 (inverse Simpson's Index). Comparisons between individuals with varying toe lesion severity (mild, moderate, severe) in top row, and individuals with differing Batrachochytrium dendrobatidis (Bd) infection status (Bd + vs Bd -) in bottom row.

\section{Sorenson Index}

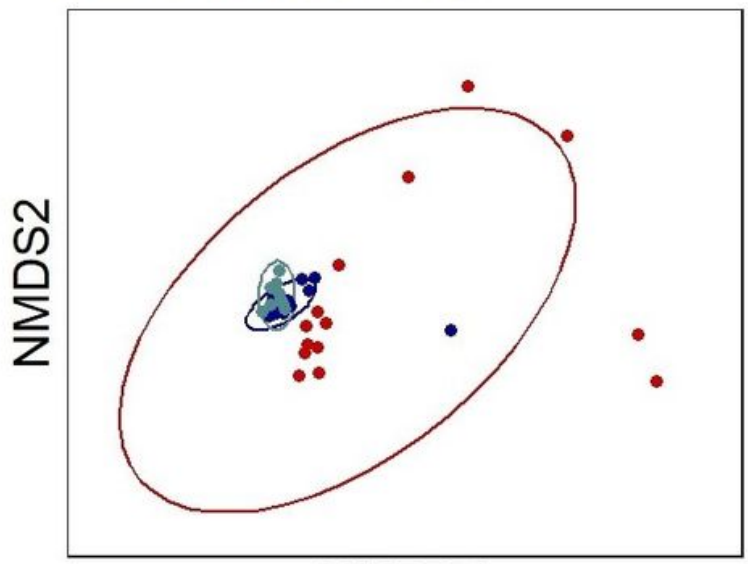

NMDS1

\section{Morisita-Horn}

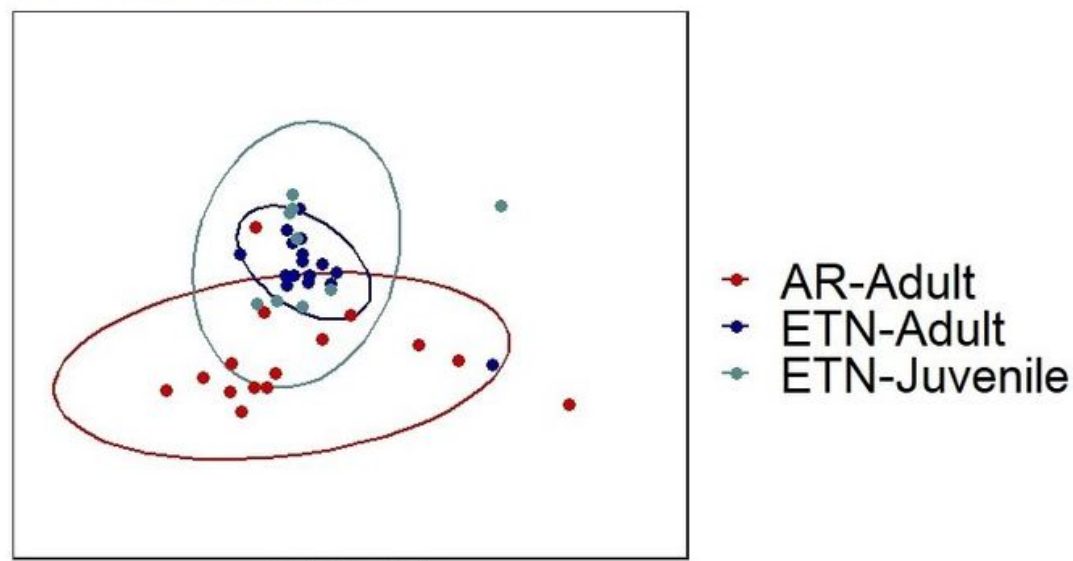

NMDS1

\section{Figure 4}

NMDS ordination plots of hellbender skin bacterial assemblages based on Sorenson and Morisita-Horn Indices. Each point represents a single skin bacterial assemblage from dorsal skin swabs. Groupings by subspecies of eastern hellbenders (Cryptobranchus alleganiensis alleganiensis) of eastern Tennessee (ETN) (blue) and Ozark hellbenders (Cryptobranchus alleganiensis bishopi) of Arkansas (red) with associated ellipses base on standard error. ETN hellbenders are further grouped by life stage of juvenile (gray-blue) and adult (dark blue).

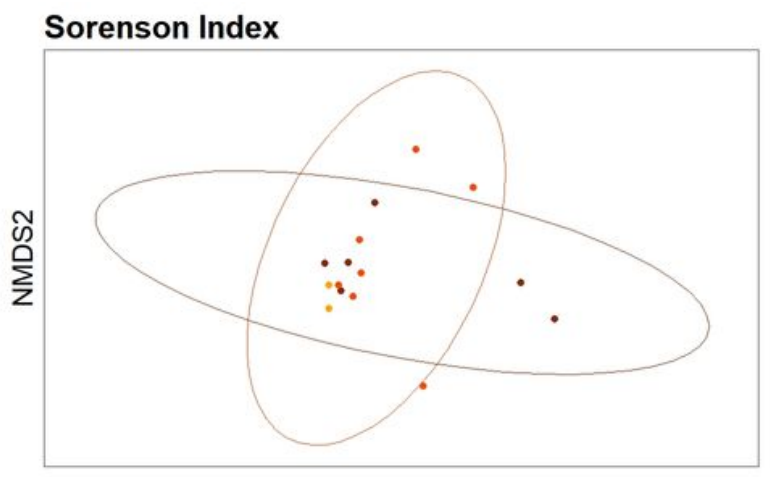

\section{Morisita-Horn}
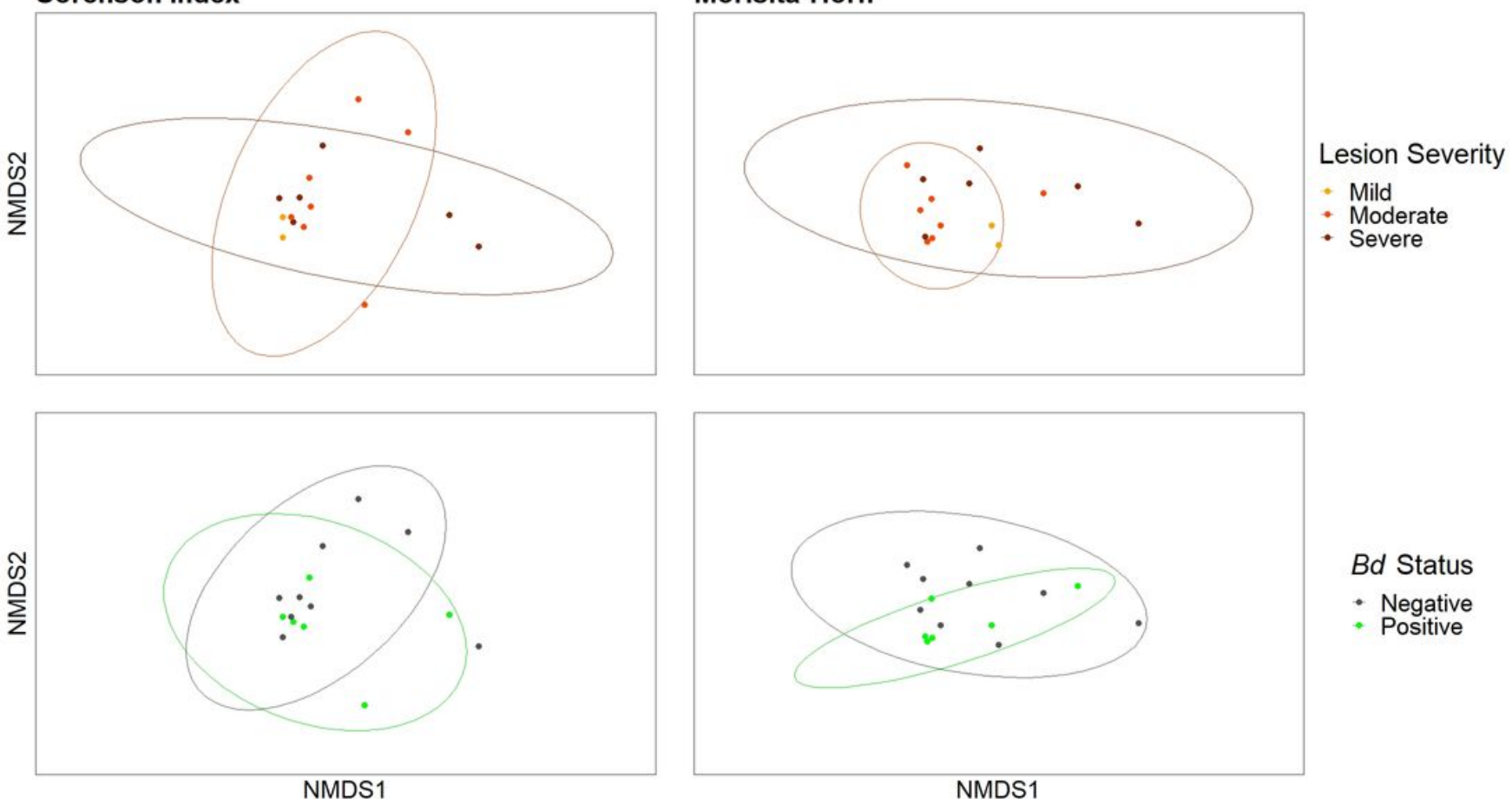

Bd Status

- Negative

- Positive

Figure 5 
NMDS ordination plots of hellbender skin bacterial assemblages based on Sorenson and Morisita-Horn Indices. Each point represents a single skin bacterial assemblage from dorsal skin swabs of Ozark hellbenders (Cryptobranchus alleganiensis bishopi) of Arkansas (AR). Top plots grouped by toe lesion severity of mild (yellow), moderate (orange), or severe (red). Bottom plots groups by Batrachochytrium dendrobatidis (Bd) infection status of $\mathrm{Bd}+(\mathrm{green})$ or $\mathrm{Bd}-$ (gray).
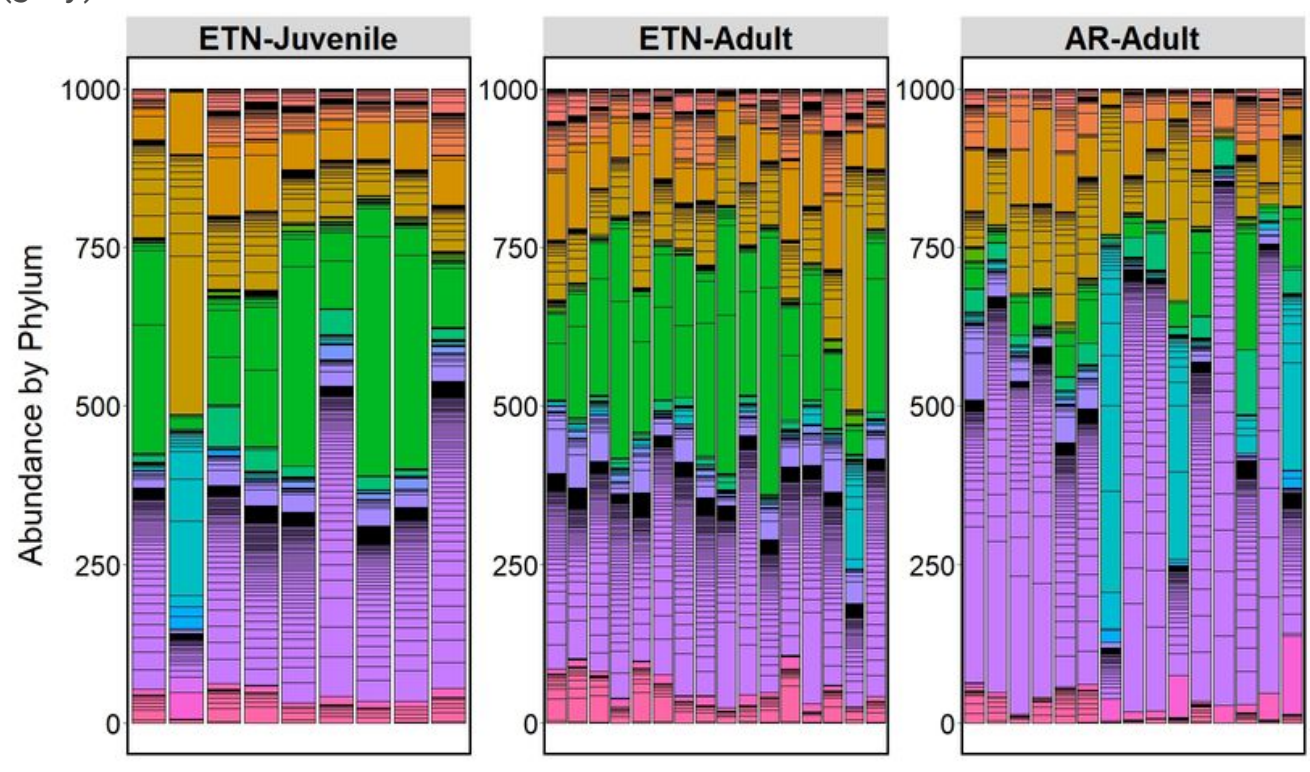

\section{Phylum}

\begin{tabular}{l|l} 
Acidobacteria & Firmicutes \\
Actinobacteria & Fusobacteria \\
Armatimonadetes & Gemmatimonadetes \\
Bacteria_unclassified & Lentisphaerae \\
Bacteroidetes & Nitrospira \\
BRC1 & OD1 \\
Chlamydiae & Planctomycetes \\
Chlorobi & Proteobacteria \\
Chloroflexi & Spirochaetes \\
Cyanobacteria_Chloroplast & SR1 \\
Deferribacteres & Synergistetes \\
Deinococcus-Thermus & TM7 \\
Elusimicrobia & Verrucomicrobia \\
Fibrobacteres & WS3
\end{tabular}

\section{Figure 6}

Phylum abundance of the 28 bacterial phlya identified from 1000 sequence subsamples of 16 S sequences obtained from dorsal skin swabs from eastern hellbenders (Cryptobranchus alleganiensis alleganiensis) captured in eastern Tennessee (ETN) and Ozark hellbenders (Cryptobranchus alleganiensis bishopi) in Arkansas (AR). ETN hellbenders are further grouped by life stage of juvenile (ETN-Juv) and adult (ETN-AR). Only adults were encountered for AR (ARAd).
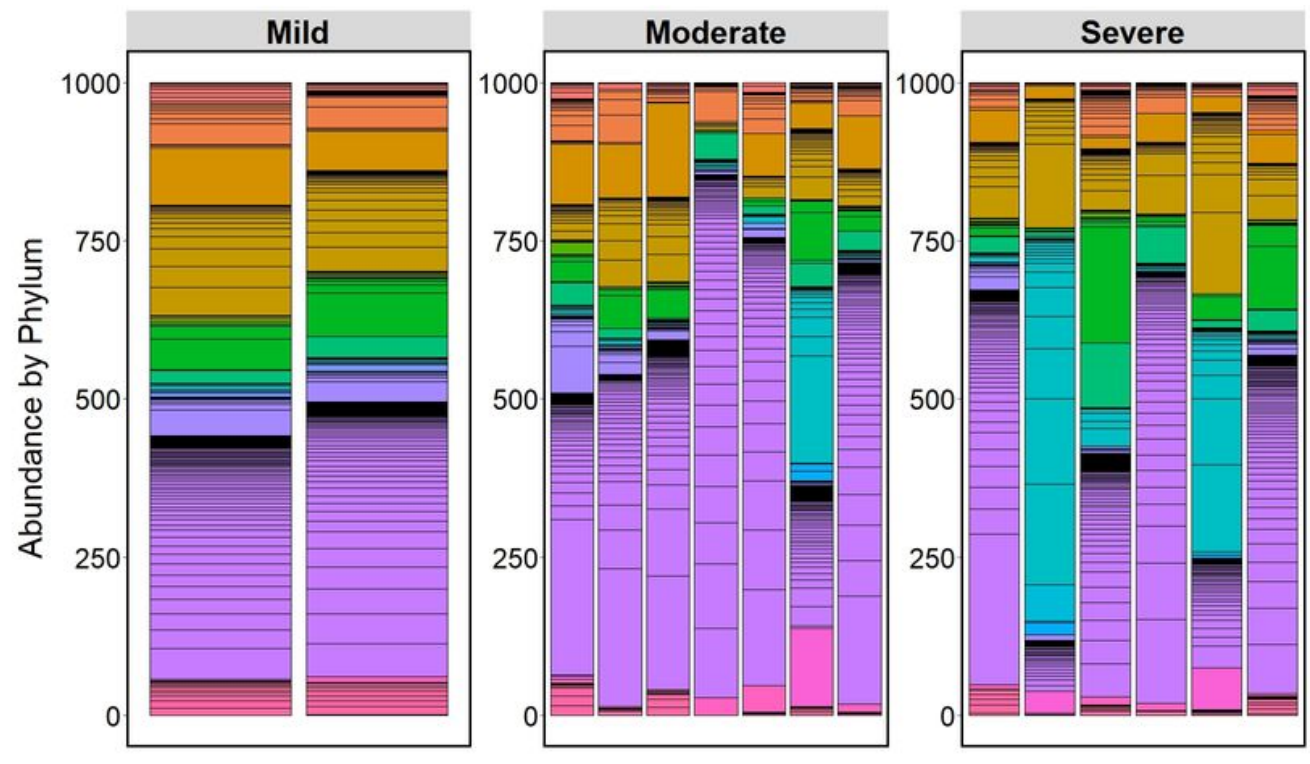

\section{Phylum}

Acidobacteria
Actinobacteria
Armatimonadetes
Bacteria_unclassified
Bacteroidetes
BRC1
Chlamydiae
Chlorobi
Chloroflexi
Cyanobacteria_Chloroplast
Deferribacteres
Deinococcus-Thermus
Elusimicrobia
Fibrobacteres

Firmicutes

Fusobacteria

Gemmatimonadetes

Lentisphaerae

Nitrospira

OD1

Planctomycetes

Proteobacteria

Spirochaetes

SR1

Synergistetes

TM7

Verrucomicrobia

WS3

\section{Figure 7}


Phylum abundance of the 28 bacterial phlya identified from 1000 sequence subsamples of $16 \mathrm{~S}$ sequences obtained from dorsal skin swabs from Ozark hellbenders (Cryptobranchus alleganiensis bishopi) captured in Arkansas (AR) grouped by toe lesion severity of mild, moderate, and severe.

\section{Supplementary Files}

This is a list of supplementary files associated with this preprint. Click to download.

- Hardmanetal.MicrobiomeHellbendersMOTHURandRCode.docx 